\title{
Homogenization in Sobolev Spaces with Nonstandard Growth: Brief Review of Methods and Applications
}

\author{
Brahim Amaziane ${ }^{1}$ and Leonid Pankratov ${ }^{1,2}$ \\ ${ }^{1}$ Laboratoire de Mathematiques et de leurs Applications, CNRS-UMR 5142, UNIV Pau \& Pays Adour, \\ Avenue de l'Université, 64000 Pau, France \\ ${ }^{2}$ Department of Mathematics, B. Verkin Institute for Low Temperature Physics and Engineering, 47, Avenue Lenin, \\ Kharkov 61103, Ukraine \\ Correspondence should be addressed to Brahim Amaziane; brahim.amaziane@univ-pau.fr
}

Received 12 July 2012; Accepted 22 January 2013

Academic Editor: Julio Rossi

Copyright (C) 2013 B. Amaziane and L. Pankratov. This is an open access article distributed under the Creative Commons Attribution License, which permits unrestricted use, distribution, and reproduction in any medium, provided the original work is properly cited.

\begin{abstract}
We review recent results on the homogenization in Sobolev spaces with variable exponents. In particular, we are dealing with the $\Gamma$-convergence of variational functionals with rapidly oscillating coefficients, the homogenization of the Dirichlet and Neumann variational problems in strongly perforated domains, as well as double porosity type problems. The growth functions also depend on the small parameter characterizing the scale of the microstructure. The homogenization results are obtained by the method of local energy characteristics. We also consider a parabolic double porosity type problem, which is studied by combining the variational homogenization approach and the two-scale convergence method. Results are illustrated with periodic examples, and the problem of stability in homogenization is discussed.
\end{abstract}

\section{Introduction}

In recent years, there has been an increasing interest in the study of the functionals with variable exponents or nonstandard $p(x)$-growth and the corresponding Sobolev spaces, see for instance [1-8] and the references therein. In particular, the conditions under which $C_{0}^{\infty}$ functions are dense in $W^{1, p(\cdot)}$ have been found. Also, Meyers estimates, which are used in the homogenization process, have been obtained in [6]. Let us mention that such partial differential equations arise in many engineering disciplines, such as electrorheological fluids, non-Newtonian fluids with thermoconvective effects, and nonlinear Darcy flow of compressible fluids in heterogeneous porous media, see for instance [1].

This paper discusses problems of homogenization in Sobolev spaces with variable exponents. Attention is focussed on the homogenization and minimization problems for variational functionals in the framework of Sobolev spaces with nonstandard growth. The material is essentially a review with some new results.
$\Gamma$-convergence and minimization problems for functionals with periodic and locally periodic rapidly oscillating Lagrangians of $p$-growth with a constant $p$ are well studied now, see for instance $[9,10]$ and the bibliography therein.

The works [11-15] (see also [16]) focus on the variational functionals with nonstandard growth conditions. In particular, the homogenization and $\Gamma$-convergence problems for Lagrangians with variable rapidly oscillating exponents $p(x)$ were considered in $[13,14]$. It was shown that the energy minimums and the homogenized Lagrangians in the spaces $W^{1, r}$ might depend on the value of $r$ (the so-called Lavrentiev phenomenon). For example, such a behavior can be observed for the Lagrangian $|\nabla u|^{p(x / \varepsilon)}$ with a periodic "chess board" exponent $p(y)$ and a small parameter $\varepsilon>0$.

Another interesting example of Lagrangian with rapidly oscillating exponent was considered in [11]. Namely, for the functional

$$
\mathscr{F}_{\varepsilon}[u]=\int|\nabla u|^{p(x / \varepsilon)} d x
$$


with a smooth periodic $p(y)$ such that $p(y)>1$, it was shown that the limit functional is bounded on Sobolev-Orlicz space of functions with gradient in an $L^{\alpha} \log$ space, where $\alpha$ is the fiber percolation level of $p(x)$.

Variational functionals with nonstandard growth conditions have also been considered in [9]. Chapter 21 of this book focuses on the $\Gamma$-convergence of such functionals in $L^{p}$ spaces.

In this paper, we are dealing mainly with the $\Gamma$-convergence of variational functionals with periodic rapidly oscillating coefficients, the homogenization of the variational problems in strongly perforated domains (Dirichlet and Neumann problems), and nonlinear double porosity type problems, that is, with the problems where the coefficient of the differential operator asymptotically degenerates on a some specially defined subset (e.g., the set of periodically distributed inclusions) of the domain under consideration. The paper is based on the results obtained in papers [17-26].

The paper is organized as follows. In Section 2, for the sake of completeness, we recall the definition and main results on the Lebesgue and Sobolev spaces with variable exponents which will be used in the sequel. Then in auxiliary Section 3, we give some definitions which will be used in the paper. In Section 4, we study the question of the $\Gamma$ convergence and homogenization of functionals with rapidly oscillating periodic coefficients. In Section 5, we are dealing with the homogenization of the Dirichlet variational problem in strongly perforated domains. The main result of the section (see Theorem 8) is then applied to the study of nonlocal effects in the homogenization (see Theorem 9). In Section 5.3, we give a periodic example, when all the conditions of Theorems 8 and 9 are satisfied and all the coefficients of the homogenized problem are calculated explicitly. Moreover, the question of stability in homogenization is also discussed here. Theorems 8 and 9 are proved by using the so-called method of local energy characteristics proposed earlier by Marchenko and Khruslov for linear homogenization problems (see [27] and the references herein). This method is close to the $\Gamma$ convergence method. Briefly, it is based on the derivation of the lim-inf and lim-sup estimates for the variational functional under consideration along with the assumptions on the behavior of the local energy characteristics. In Section 6, the homogenization of the Neumann problem in strongly perforated domains is considered. In this section, the closeness of the method of local energy characteristics is shown directly. In Section 7, we are dealing with a variational problem with high contrast coefficients (nonlinear double porosity type model). The main result of the section (Theorem 23) is also obtained by the method of local energy characteristics. As an application of this result, we consider the periodic case, where we focus our attention on the question of stability in homogenization. Finally, in Section 8, we are dealing with the homogenization of a class of quasilinear parabolic equations with nonstandard growth. The main results of the section are obtained by combining the two-scale convergence method and the variational homogenization approach.

\section{Sobolev Spaces with Variable Exponents}

In this section, we introduce the function spaces used throughout the paper and describe their basic properties. We refer here to $[1,4,5,7,8]$.

Let $\Omega$ be a bounded Lipschitz domain in $\mathbb{R}^{n}(n \geq 2)$. We introduce the function $p=p(x)$ and assume that this function is bounded such that

$$
1<p^{-}=\inf _{\Omega} p(x) \leq p(x) \leq \sup _{\Omega} p(x)=p^{+}<+\infty .
$$

We also assume that the function $p(x)$ satisfies the log-Hölder continuity property. Namely, for all $x, y \in \Omega$,

$$
|p(x)-p(y)| \leq \omega(|x-y|) \text { with } \varlimsup_{\tau \rightarrow 0} \omega(\tau) \ln \left(\frac{1}{\tau}\right) \leq C .
$$

Notice that this property was introduced by Zhikov to avoid the so-called Lavrentiev phenomenon (see, e.g., [15]).

(1) By $L^{p(\cdot)}(\Omega)$ we denote the space of measurable functions $f$ in $\Omega$ such that

$$
A_{p(\cdot), \Omega}(f)=\int_{\Omega}|f(x)|^{p(x)} d x<+\infty .
$$

The space $L^{p(\cdot)}(\Omega)$ equipped with the norm

$$
\|f\|_{L^{p(\cdot)}(\Omega)}=\inf \left\{\lambda>0: A_{p(\cdot), \Omega}\left(\frac{f}{\lambda}\right) \leq 1\right\}
$$

becomes a Banach space.

(2) The following inequalities hold:

$$
\begin{aligned}
& \min \left(\|f\|_{L^{p(\cdot)}(\Omega)}^{p^{-}},\|f\|_{L^{p^{(\cdot)}(\Omega)}}^{p^{+}}\right) \\
& \quad \leq A_{p(\cdot), \Omega}(f) \\
& \quad \leq \max \left(\|f\|_{L^{p \cdot()}(\Omega)}^{p^{-}},\|f\|_{L^{p^{(\cdot)}(\Omega)}}^{p^{+}}\right), \\
& \min \left(A_{p(\cdot), \Omega}^{1 / p^{-}}, A_{p(\cdot), \Omega}^{1 / p^{+}}\right) \\
& \quad \leq\|f\|_{L^{p(\cdot)}(\Omega)} \\
& \quad \leq \max \left(A_{p(\cdot), \Omega}^{1 / p^{-}}, A_{p(\cdot), \Omega}^{1 / p^{+}}\right) .
\end{aligned}
$$

(3) Let $f \in L^{p(\cdot)}(\Omega), g \in L^{q(\cdot)}(\Omega)$ with

$$
\begin{aligned}
\frac{1}{p(x)}+\frac{1}{q(x)}=1, & 1<p^{-} \leq p(x) \leq p^{+}<\infty, \\
& 1<q^{-} \leq q(x) \leq q^{+}<+\infty .
\end{aligned}
$$

Then the Hölder's inequality holds

$$
\int_{\Omega}|f g| d x \leq 2\|f\|_{L^{p(\cdot)}(\Omega)}\|g\|_{L^{q(\cdot)}(\Omega)} .
$$


(4) According to (8), for every $1 \leq q=$ const $<p^{-} \leq$ $p(x)<+\infty$

$$
\|f\|_{L^{q}(\Omega)} \leq C\|f\|_{L^{p(\cdot)}(\Omega)}
$$

with the constant $C=2\|1\|_{p(\cdot) /\left(L^{p(\cdot)-q}(\Omega)\right)}$.

It is straightforward to check that for domains $\Omega$ such that meas $\Omega<+\infty$,

$\|1\|_{L^{p(\cdot)}(\Omega)} \leq 2 \max \left\{[\text { meas } \Omega]^{2 / p^{-}},[\text {meas } \Omega]^{1 / 2 p^{+}}\right\}$.

(5) The space $\left.W^{1, p(\cdot)}(\Omega), p(\cdot) \in\left[p^{-}, p^{+}\right] \subset\right] 1,+\infty[$, is defined by

$W^{1, p(\cdot)}(\Omega)=\left\{f \in L^{p^{(\cdot)}}(\Omega):|\nabla f| \in L^{p^{(\cdot)}}(\Omega)\right\}$.

If condition (3) is satisfied, $W_{0}^{1, p(\cdot)}(\Omega)$ is the closure of the set $C_{0}^{\infty}(\Omega)$ with respect to the norm of $W^{1, p(\cdot)}(\Omega)$. If the boundary of $\Omega$ is Lipschitz continuous and $p(x)$ satisfies (3), then $C_{0}^{\infty}(\Omega)$ is dense in $W_{0}^{1, p(\cdot)}(\Omega)$. The norm in the space $W_{0}^{1, p(\cdot)}$ is defined by

$$
\|u\|_{W_{0}^{1, p(\cdot)}}=\sum_{i}\left\|D_{i} u\right\|_{L^{p(\cdot)}(\Omega)}+\|u\|_{L^{p(\cdot)}(\Omega)}
$$

If the boundary of $\Omega$ is Lipschitz and $p \in C^{0}(\Omega)$, then the norm $\|\cdot\|_{W_{0}^{1, p(\cdot)}(\Omega)}$ is equivalent to the norm

$$
{\widetilde{\|u\|_{W_{0}^{1, p(x)}(\Omega)}}}=\sum_{i}\left\|D_{i} u\right\|_{L^{p(\cdot)}(\Omega)}
$$

(6) If $p \in C^{0}(\bar{\Omega})$, then $W^{1, p(\cdot)}(\Omega)$ is separable and reflexive.

(7) If $p, q \in C^{0}(\bar{\Omega})$,

$p_{*}(x)$

$=\left\{\begin{array}{lll}\frac{p(x) n}{n-p(x)} & \text { if } p(x)<n, & \\ & & \text { and } 1<q(x) \leq \sup _{\Omega} q(x) \\ +\infty & \text { if } p(x)>n, & <\inf _{\Omega} p_{*}(x),\end{array}\right.$

then the embedding $W_{0}^{1, p(\cdot)}(\Omega) \hookrightarrow L^{q(\cdot)}(\Omega)$ is continuous and compact.

(8) Friedrich's inequality is valid in the following form: if $p(x)$ satisfies conditions (2) and (3), then there exists a constant $C>0$ such that for every $f \in W_{0}^{1, p(\cdot)}(\Omega)$

$$
\|f\|_{L^{p(\cdot)}(\Omega)} \leq C\|\nabla f\|_{L^{p(\cdot)}(\Omega)} .
$$

\section{Definitions}

In this auxiliary section, we introduce the necessary definitions that will be used in the paper. We start by introducing the class of the variable exponents $p_{\varepsilon}$, where $\varepsilon$ is a small positive parameter characterizing the microscopic length scale.

Definition 1 (class $\mathfrak{Q}_{p_{0}(\cdot)}^{\varepsilon}$ ). A sequence of functions $\left\{p_{\varepsilon}\right\}_{(\varepsilon>0)}$ is said to belong to the class $\mathfrak{Q}_{p_{0}(\cdot)}^{\varepsilon}$ if this sequence possesses the following properties:

(i) for any $\varepsilon>0, p_{\varepsilon}$ is bounded in the following sense:

$$
\begin{aligned}
1<p^{-} \leq p_{\varepsilon}^{-} & \equiv \min _{x \in \bar{\Omega}} p_{\varepsilon}(x) \leq p_{\varepsilon}(x) \leq \max _{x \in \bar{\Omega}} p_{\varepsilon}(x) \\
& \equiv p_{\varepsilon}^{+} \leq p^{+}<+\infty \quad \text { in } \bar{\Omega}
\end{aligned}
$$

(ii) for any $\varepsilon>0, p_{\varepsilon}$ satisfies the log-Hölder continuity property;

(iii) the function $p_{\varepsilon}$ converges uniformly in $\Omega$ to a function $p_{0}$, where the limit function $p_{0}$ is assumed to satisfy the log-Hölder continuity property.

We also recall the definition of the $\Gamma$-convergence (see, e.g., $[9,10,28]$ and the bibliography therein). In our case this definition takes the following form.

Definition $2\left(\Gamma_{p(\cdot)}\right.$-convergence). The functional $I^{\varepsilon}: L^{p_{\varepsilon}(\cdot)}$ $(\Omega) \rightarrow \mathbb{R} \cup\{\infty\}$ is said to $\Gamma_{p_{0}(\cdot)}$-converge to a functional $I: L^{p_{0}(\cdot)}(\Omega) \rightarrow \mathbb{R} \cup\{\infty\}$ if

(a) ("lim inf"-inequality) for any $u \in L^{p_{0}(\cdot)}(\Omega)$ and any sequence $\left\{u^{\varepsilon}\right\}_{\mathcal{E}>0} \subset L^{p_{0}(\cdot)}(\Omega)$ which converges to the function $u$ strongly in the space $L^{p_{0}(\cdot)}(\Omega)$ we have

$$
\underline{\lim }_{\varepsilon \rightarrow 0} I^{\varepsilon}\left[u^{\varepsilon}\right] \geq I[u]
$$

(b) ("lim sup"-inequality) for any $u \in L^{p_{0}(\cdot)}(\Omega)$, there exists a sequence $\left\{w^{\varepsilon}\right\}_{\mathcal{E}>0} \subset L^{p_{0}(\cdot)}(\Omega)$ such that $\left\{w^{\varepsilon}\right\}_{\mathcal{E}>0}$ converges to the function $u(\cdot)$ strongly in the space $L^{p_{0}(\cdot)}(\Omega)$, and

$$
\varlimsup_{\varepsilon \rightarrow 0} I^{\varepsilon}\left[w^{\varepsilon}\right] \leq I[u]
$$

We define the strong convergence in $L^{p_{0}(\cdot)}\left(\Omega^{\varepsilon}\right)$ in the following way.

Definition 3 (strong convergence in $\left.L^{p_{0}(\cdot)}\left(\Omega^{\varepsilon}\right)\right)$. The sequence $\left\{\omega^{\varepsilon}\right\}_{\mathcal{E}>0} \in L^{p_{0}(\cdot)}\left(\Omega^{\varepsilon}\right)$ is said to converge strongly in the space $L^{p_{0}(\cdot)}\left(\Omega^{\varepsilon}\right)$ to a function $\omega \in L^{p_{0}(\cdot)}(\Omega)$ if

$$
\lim _{\varepsilon \rightarrow 0}\left\|\omega^{\varepsilon}-\omega\right\|_{L^{p_{0}(\cdot)}\left(\Omega^{\varepsilon}\right)}=0
$$

Finally, we recall the definition of the two-scale convergence (see, e.g., [29]). 
Definition 4. Let $Y=] 0,1\left[^{n}\right.$ be a basic cell and $\Omega_{T}=$ $\Omega \times] 0, T$. A function, $\varphi \in L^{2}\left(\Omega_{T} ; C_{\#}^{\infty}(Y)\right)$, which is $Y$ periodic in $y$ and which satisfies

$$
\lim _{\varepsilon \rightarrow 0} \int_{\Omega_{T}}\left|\varphi\left(x, \frac{x}{\varepsilon}, t\right)\right|^{2} d x d t=\int_{\Omega_{T} \times Y}|\varphi(x, y, t)|^{2} d y d x d t
$$

is called an admissible test function.

Definition 5 (two-scale convergence). A sequence of functions $v^{\varepsilon} \in L^{2}\left(\Omega_{T}\right)$ two-scale converges to $v \in L^{2}\left(\Omega_{T} \times Y\right)$ if for any admissible test function $\varphi(x, y, t)$,

$$
\begin{aligned}
\lim _{\varepsilon \rightarrow 0} \int_{\Omega_{T}} v^{\varepsilon}(x, t) \varphi\left(x, \frac{x}{\varepsilon}, t\right) d x d t \\
\quad=\int_{\Omega_{T} \times Y} v(x, y, t) \varphi(x, y, t) d y d x d t .
\end{aligned}
$$

This convergence is denoted by $v^{\varepsilon}(x, t) \stackrel{2 s}{\rightarrow} v(x, y, t)$.

The method of the local energy characteristics, generally speaking, deals with nonperiodic structures. We often make use of the following definition.

Definition 6 (distribution in an asymptotically regular way). The set $\mathscr{F}^{\mathcal{E}}$ is said to be distributed in an asymptotically regular way in $\Omega$, if for any ball $V(y, r)$ of radius $r$ centered at $y \in \Omega$ and $\varepsilon>0$ small enough $\left(\varepsilon \leq \varepsilon_{0}(r)\right), V(y, r) \cap \mathscr{F}^{\varepsilon} \neq \emptyset$ and $V(y, r) \cap\left(\Omega \backslash \mathscr{F}^{\varepsilon}\right) \neq \emptyset$.

\section{4. $\Gamma$-Convergence and Homogenization of Functionals with Rapidly Oscillating Coefficients in Sobolev Spaces with Variable Exponents}

Let $\Omega$ be a bounded domain in $\mathbb{R}^{n}(n \geq 2)$ with a sufficiently smooth boundary and denote that $Y=] 0,1{ }^{n}$. We assume that a family of continuous functions $\left\{p_{\varepsilon}\right\}_{(\varepsilon>0)}$ belongs to the class, $\mathbf{Q}_{p_{0}(\cdot)}^{\varepsilon}$, and we also suppose that

(H.1) $a=a(y)$ and $b=b(y)$ are $Y$-periodic measurable functions such that

$$
0<a_{0} \leq a(y) \leq a_{1}, \quad 0<b_{0} \leq b(y) \leq b_{1} .
$$

(H.2) $f \in C(\Omega)$.

For the notational convenience, we set

$$
\begin{aligned}
& a_{p_{\varepsilon}}^{\varepsilon}(x)=\frac{1}{p_{\varepsilon}(x)} a\left(\frac{x}{\varepsilon}\right), \\
& b_{p_{\varepsilon}}^{\varepsilon}(x)=\frac{1}{p_{\varepsilon}(x)} b\left(\frac{x}{\varepsilon}\right) .
\end{aligned}
$$

In the space $L^{p_{\varepsilon}(\cdot)}(\Omega)$, we define the functional $J^{\varepsilon}: L^{p_{\varepsilon}(\cdot)}$ $(\Omega) \rightarrow \mathbb{R}:$

$J^{\varepsilon}[u]$

$$
\stackrel{\text { def }}{=}\left\{\begin{array}{cl}
\int_{\Omega}\left\{a_{p_{\varepsilon}}^{\varepsilon}(x)|\nabla u|^{p_{\varepsilon}(x)}+b_{p_{\varepsilon}}^{\varepsilon}(x)|u|^{p_{\varepsilon}(x)}\right. & \\
-f(x) u\} d x, & \text { if } u \in W^{1, p_{\varepsilon}(\cdot)}(\Omega), \\
+\infty, & \text { otherwise. }
\end{array}\right.
$$

We study the asymptotic behavior of $J^{\varepsilon}$ and its minimizers as $\varepsilon \rightarrow 0$. Our analysis relies on the $\Gamma$-convergence approach in Sobolev spaces with variable exponents. The main result of the section is the following.

Theorem 7. Let assumptions (H.1) and (H.2) be fulfilled. Then

(i) The functional $J^{\varepsilon}, \Gamma_{p_{0}(\cdot)}$-converges to the functional $J_{\text {hom }}: L^{p_{0}(\cdot)}(\Omega) \rightarrow \mathbb{R} \cup\{+\infty\}$ given by

$J_{\text {hom }}[u]$

$$
=\left\{\begin{array}{cl}
\int_{\Omega}\left\{\mathrm{T}(x, \nabla u)+\beta_{p}(x)|u|^{p_{0}(x)}\right. & \\
-f(x) u\} d x, & \text { if } u \in W^{1, p_{0}}(\Omega), \\
+\infty, & \text { otherwise, }
\end{array}\right.
$$

where

$\mathrm{T}(x, \mathbf{b})$

$$
=\inf \left\{\frac{1}{p_{0}(x)} \int_{Y} a(y) \mid \nabla v(y)\right.
$$

$$
\left.+\left.\mathbf{b}\right|^{p_{0}(x)} d y: v \in W_{\#}^{1, p_{0}(x)}(Y)\right\},
$$

$\beta_{p}(x)$

$$
=\frac{1}{p_{0}(x)} \int_{Y} b(y) d y .
$$

(ii) The minimizer $u^{\varepsilon}$ of the functional $J^{\varepsilon}$ converges to the minimizer $u$ of the functional $J_{\text {hom }}$ strongly in the space $L^{p_{0}(\cdot)}(\Omega)$.

The Scheme of the Proof of Theorem 7 (See [18] for More Details) Is as Follows. We start our analysis by proving the "lim inf"-inequality. The proof of this inequality is done in two main steps. First we introduce an auxiliary functional $\widetilde{J}^{\varepsilon}: L^{\pi_{\varepsilon}(\cdot)}(\Omega) \rightarrow \mathbb{R}:$

$\widetilde{J}^{\varepsilon}[u]$

$$
=\left\{\begin{array}{cl}
\int_{\Omega}\left\{a_{p_{\varepsilon}}^{\varepsilon}(x)|\nabla u|^{\pi_{\varepsilon}(x)}+b_{p_{\varepsilon}}^{\varepsilon}(x)|u|^{p_{\varepsilon}(x)}\right. & \\
-f(x) u\} d x, & \text { if } u \in W^{1, \pi_{\varepsilon}(\cdot)}(\Omega) ; \\
+\infty, & \text { otherwise, }
\end{array}\right.
$$


where $\pi_{\varepsilon}(x)=\min \left\{p_{\varepsilon}(x), p_{0}(x)\right\}$ and prove the "lim inf"inequality for this functional. Then, at the second step, we show that the "lim inf"-inequality for the auxiliary functional $\widetilde{J}^{\varepsilon}$ implies the "lim inf"-inequality for $J^{\varepsilon}$. Then, using a special test function and the Meyers estimate (see [6]), we obtain the "lim sup"-inequality. Finally, we prove the convergence of the minimizers. This completes the proof of Theorem 7.

\section{Homogenization of the Dirichlet Problem and Related Questions}

5.1. Homogenization of the Dirichlet Problem. Let $\Omega$ be a bounded domain in $\mathbb{R}^{n}(n \geq 2)$ with sufficiently smooth boundary. Let $\mathscr{F}^{\varepsilon}$ be an open subset in $\Omega$. Here $\varepsilon$ is a small parameter characterizing the scale of the microstructure. We assume that $\mathscr{F}^{\varepsilon}$ is distributed in an asymptotically regular way in $\Omega$ and we set

$$
\Omega^{\varepsilon}=\Omega \backslash \overline{\mathscr{F}}^{\varepsilon} .
$$

Let $p_{\varepsilon}=p_{\varepsilon}(x)$ be a continuous function defined in the domain $\bar{\Omega}$. We assume that, for any $\varepsilon>0$, it satisfies the following conditions:

(A.1.1) the function $p_{\varepsilon}(x)$ is bounded in the following sense:

$$
\begin{aligned}
1<p^{-} \leq p_{\varepsilon}^{-} & \equiv \min _{x \in \bar{\Omega}} p_{\varepsilon}(x) \leq p_{\varepsilon}(x) \leq \max _{x \in \bar{\Omega}} p_{\varepsilon}(x) \\
& \equiv p_{\varepsilon}^{+} \leq p^{+} \leq n \quad \text { in } \bar{\Omega} ;
\end{aligned}
$$

(A.1.2) the function $p_{\varepsilon}$ satisfies the log-Hölder continuity property;

(A.1.3) the function $p_{\varepsilon}$ converges uniformly in $\Omega$ to a function $p_{0}$, where the limit function $p_{0}$ is assumed to satisfy the log-Hölder continuity property;

(A.1.4) the function $p_{\varepsilon}$ is such that $p_{\varepsilon}(x) \geq p_{0}(x)$ in $\Omega$.

We consider the following variational problem:

$$
J^{\varepsilon}[u] \stackrel{\text { def }}{=} \int_{\Omega^{\varepsilon}} F_{\varepsilon}(x, u, \nabla u) d x \longrightarrow \min , \quad u \in W_{0}^{1, p_{\varepsilon}(\cdot)}\left(\Omega^{\varepsilon}\right),
$$

where

$$
\mathrm{F}_{\varepsilon}(x, u, \nabla u)=\frac{1}{p_{\varepsilon}(x)}|\nabla u|^{p_{\varepsilon}(x)}+\frac{1}{p_{\varepsilon}(x)}|u|^{p_{\varepsilon}(x)}-f(x) u,
$$

and $f \in C^{1}(\Omega)$. It is known from $[1-3,6]$ that for each $\varepsilon>0$, there exists a unique solution (minimizer) $u^{\varepsilon} \in W^{1, p_{\varepsilon}(\cdot)}\left(\Omega^{\varepsilon}\right)$ of problem (30). Let us extend $u^{\varepsilon}$ in $\mathscr{F}^{\varepsilon}$ by zero (keeping for it the same notation). Then we obtain the family $\left\{u^{\varepsilon}\right\} \subset$ $W^{1, p_{\varepsilon}(\cdot)}(\Omega)$. We study the asymptotic behavior of $u^{\varepsilon}$ as $\varepsilon \rightarrow$ 0 .

Instead of the classical periodicity assumption on the microstructure of the perforated domain $\Omega^{\varepsilon}$, we impose certain conditions on the local energy characteristic of the set
$\mathscr{F}^{\varepsilon}$. To this end we introduce $K_{h}^{z}$ an open cube centered at $z \in \Omega$ with length equal to $h(0<\varepsilon \ll h<1)$, and we set

$$
\begin{aligned}
c^{\varepsilon, h} & (z, b) \\
& =\inf _{v^{\varepsilon}} \int_{K_{h}^{z}}\left\{\frac{1}{p_{\varepsilon}(x)}\left|\nabla v^{\varepsilon}\right|^{p_{\varepsilon}(x)}+h^{-p^{+}-\gamma}\left|v^{\varepsilon}-b\right|^{p_{\varepsilon}(x)}\right\} d x,
\end{aligned}
$$

where $\gamma>0$, and the infimum is taken over $v^{\varepsilon} \in W^{1, p_{\varepsilon}(\cdot)}(\Omega)$ that equal zero in $\mathscr{F}^{\varepsilon}$. We assume that

(C.1.1) there exists a continuous function $c(x, b)$ such that for any $x \in \Omega$, any $b \in \mathbb{R}$, and a certain $\gamma=\gamma_{0}>0$,

$$
\lim _{h \rightarrow 0} \varlimsup_{\varepsilon \rightarrow 0} h^{-n} c^{\varepsilon, h}(z, b)=\lim _{h \rightarrow 0} \frac{\lim _{\varepsilon \rightarrow 0}}{h^{-n}} c^{\varepsilon, h}(z, b)=c(x, b) ;
$$

(C.1.2) there exists a constant $A$ independent of $\varepsilon$ such that for any $x \in \Omega$,

$$
\lim _{h \rightarrow 0} \varlimsup_{\varepsilon \rightarrow 0} h^{-n} c^{\varepsilon, h}(z, b) \leq A\left(1+|b|^{p_{0}(x)}\right) .
$$

The first main result of Section 5 is the following.

Theorem 8. Let conditions (A.1.1)-(A.1.4) on the function $p_{\varepsilon}$ and conditions (C.1.1) and (C.1.2) on the local characteristic be satisfied. Then $u^{\varepsilon}$ the solution (minimizer) of the variational problem (30) (extended by zero in $\mathscr{F}^{\varepsilon}$ ) converges weakly in $W^{1, p_{0}(\cdot)}(\Omega)$ to $u$ the solution (minimizer) of

$$
\begin{array}{r}
J_{\text {hom }}[u] \stackrel{\text { def }}{=} \int_{\Omega}\left\{F_{0}(x, u, \nabla u)+c(x, u)-f(x) u\right\} d x \longrightarrow \min , \\
u \in W_{0}^{1, p_{0}(\cdot)}(\Omega),
\end{array}
$$

where

$$
\mathrm{F}_{0}(x, w, \nabla w) \stackrel{\text { def }}{=} \frac{1}{p_{0}(x)}|\nabla w|^{p_{0}(x)}+\frac{1}{p_{0}(x)}|w|^{p_{0}(x)}-g(x) w .
$$

The Scheme of the Proof of Theorem 8 (See [19] for More Details) Is as Follows. First, it follows from (6), (30), and the regularity properties of the functions $f, p_{\varepsilon}$ that $\left\|{u^{\varepsilon}}^{\varepsilon}\right\|_{W^{1, p_{\varepsilon}(\cdot)}\left(\Omega^{\varepsilon}\right)} \leq C$. We extend $u^{\varepsilon}$ by zero to the set $\mathscr{F}^{\varepsilon}$ and consider $\left\{u^{\varepsilon}\right\}$ as a sequence in $W^{1, p_{\varepsilon}(\cdot)}(\Omega)$. Then $\left\|u^{\varepsilon}\right\|_{W^{1, p_{\varepsilon}(\cdot)}(\Omega)} \leq$ C. Condition (A.1.4) immediately implies that

$$
\left\|u^{\varepsilon}\right\|_{W^{1, p_{0}(\cdot)}(\Omega)} \leq C .
$$

Hence, one can extract a subsequence $\left\{u^{\varepsilon}, \varepsilon=\varepsilon_{k} \rightarrow 0\right\}$ that converges weakly to a function $u \in W^{1, p_{0}(\cdot)}(\Omega)$. We will show that $u$ is the solution of the variational problem (35). The proof is done in two mains steps. On the first step, we prove the "lim sup"-inequality (the upper bound for the functional $\left.J^{\varepsilon}\right)$. To this end, we cover the domain $\Omega$ by the cubes $K_{h}^{\alpha}$ of length $h>0$ centered at the points $x^{\alpha}$, where $\left\{x^{\alpha}\right\}$ be a 
periodic grid in $\Omega$ with a period $h^{\prime}=h-h^{1+\gamma / p^{+}}(\varepsilon \ll h \ll 1$, $\left.0<\gamma<p^{+}\right)$. We associate with this covering a partition of unity $\left\{\varphi_{\alpha}\right\}: 0 \leq \varphi_{\alpha}(x) \leq 1 ; \varphi_{\alpha}(x)=0$ for $x \notin K_{h}^{\alpha}$; $\varphi_{\alpha}(x)=1$ for $x \in K_{h}^{\alpha} \backslash \cup_{\beta \neq \alpha} K_{h}^{\beta} ; \sum_{\alpha} \varphi_{\alpha}(x)=1$ for $x \in \Omega$; $\left|\nabla \varphi_{\alpha}(x)\right| \leq C h^{-1-\gamma / p^{+}}$. Then we construct the function $w_{h}^{\varepsilon}$ using the minimizers $v_{\alpha}^{\varepsilon}=v_{\alpha}^{\varepsilon}(x)$ of (32) for $z=x^{\alpha}$. We show that, for any $w \in W_{0}^{1, p_{0}(\cdot)}(\Omega)$,

$$
\lim _{h \rightarrow 0} \varlimsup_{\varepsilon \rightarrow 0} J^{\varepsilon}\left[w_{h}^{\varepsilon}\right] \leq J_{\text {hom }}[w]
$$

and, therefore,

$$
\varlimsup_{\varepsilon \rightarrow 0} J^{\varepsilon}\left[u^{\varepsilon}\right] \leq J_{\text {hom }}[w]
$$

On the second step, we prove the "lim inf"-inequality (the lower bound for the functional $J^{\varepsilon}$ ) using the definition of the local energy characteristic and the convexity of our functional:

$$
\varliminf_{\varepsilon=\overline{\varepsilon_{k} \rightarrow 0}} J^{\varepsilon}\left[u^{\varepsilon}\right] \geq J_{\text {hom }}[u] .
$$

Finally it follows from (39) and (40) that

$$
J_{\text {hom }}[u] \leq J_{\text {hom }}[w] \text {, }
$$

for any $w \in W^{1, p_{0}(\cdot)}(\Omega)$ such that $w=0$ on $\partial \Omega$. This means that any weak limit of the solution of problem (30) extended to the set $\mathscr{F}^{\varepsilon}$ by zero is the solution of the homogenized problem (35). This completes the proof of Theorem 8 . The generalization of Theorem 8 is given in [17].

5.2. Nonlocal Effects in Homogenization of $p_{\varepsilon}(x)$-Laplacian in Perforated Domains. Let $\Omega$ be a domain in $\mathbb{R}^{n}(n \geq 3)$ defined in the previous section. Let $\mathscr{F}^{\varepsilon}$ be an open connected subset in $\Omega$ like a net. We assume that the set $\mathscr{F}^{\varepsilon}$ satisfies the following conditions:

(F.1.1) $\mathscr{F}^{\varepsilon} \Subset \Omega_{d}$, where $\Omega_{d}=\{x \in \Omega: \operatorname{dist}(x, \partial \Omega) \geq d\}$ with $d>0$ independent of $\varepsilon$;

(F.1.2) $\mathscr{F}^{\varepsilon}$ is distributed in an asymptotically regular way in $\Omega_{d}$;

(F.1.3) the subdomain $\Omega^{\varepsilon} \stackrel{\text { def }}{=} \Omega \backslash \overline{\mathscr{F}^{\varepsilon}}$ is a connected set.

We assume that for any $\varepsilon>0$, the function $p_{\varepsilon}=$ $p_{\varepsilon}(x)$ satisfies the conditions (A.1.1)-(A.1.4) from Section 5.1. In the space $W^{1, p_{\varepsilon}(\cdot)}\left(\Omega^{\varepsilon}\right)$, we define the functional $J^{\varepsilon}$ : $W^{1, p_{\varepsilon}(\cdot)}\left(\Omega^{\varepsilon}\right) \rightarrow \mathbb{R}$

$$
J^{\varepsilon}[u]= \begin{cases}\int_{\Omega^{\varepsilon}} F_{\varepsilon}(x, u, \nabla u) d x & \text { if } u \in W^{1, p_{\varepsilon}(\cdot)}\left(\Omega^{\varepsilon}\right) \\ +\infty & \text { otherwise }\end{cases}
$$

where $\mathrm{F}_{\varepsilon}(x, u, \nabla u)$ is defined in (31) and we consider the following variational problem:

$$
\begin{aligned}
& J^{\varepsilon}\left[u^{\varepsilon}\right] \longrightarrow \text { inf, } \quad u^{\varepsilon} \in W^{1, p_{\varepsilon}(\cdot)}\left(\Omega^{\varepsilon}\right) ; \\
& u^{\varepsilon}=A^{\varepsilon} \text { on } \partial \mathscr{F}^{\varepsilon}, \quad u^{\varepsilon}=0 \quad \text { on } \partial \Omega,
\end{aligned}
$$

where $A^{\varepsilon}$ is an unknown constant. It is known from $[1,2]$ that for each $\varepsilon>0$, there exists a unique solution $u^{\varepsilon} \in W^{1, p_{\varepsilon}(\cdot)}\left(\Omega^{\varepsilon}\right)$ of the variational problem (43). We extend $u^{\varepsilon}$ by the equality $u^{\varepsilon}=A^{\varepsilon}$ in $\mathscr{F}^{\varepsilon}$ and keep for it the same notation. Thus, we obtain the family $\left\{u^{\varepsilon}\right\}_{(\varepsilon>0)} \subset W^{1, p_{\varepsilon}(\cdot)}(\Omega)$. We study the asymptotic behavior of the family $\left\{u^{\varepsilon}\right\}_{(\varepsilon>0)}$ as $\varepsilon \rightarrow 0$.

Let us introduce the functional $J_{\text {hom }}: W^{1, p_{0}(\cdot)}(\Omega) \rightarrow \mathbb{R}$,

$$
\begin{aligned}
& J_{\text {hom }}[u, A] \\
& = \begin{cases}\int_{\Omega}\left\{\mathrm{F}_{0}(x, u, \nabla u)\right. \\
\left.+\mathbf{1}_{d}(x) c(x, u-A) d x\right\} & \text { if } u \in W^{1, p_{0}(\cdot)}(\Omega), \\
+\infty & \text { otherwise, }\end{cases}
\end{aligned}
$$

where $\mathbf{1}_{d}=\mathbf{1}_{d}(x)$ is the characteristic function of the domain $\Omega_{d}$ and $\mathrm{F}_{0}(x, u, \nabla u)$ is defined in (36).

The main result of the section is the following.

Theorem 9. Let $u^{\varepsilon}$ be a solution of (43) extended by the equality $u^{\varepsilon}(x)=A^{\varepsilon}$ in $\mathscr{F}^{\varepsilon}$. Assume that the conditions (F.1.1)(F.1.3), (A.1.1)-(A.1.4), and(C.1.1)-(C.1.2) on the set $\mathscr{F}^{\varepsilon}$, the functions $p_{\varepsilon}$, and the local characteristic $c^{\varepsilon, h}(x, b)$ are satisfied. Then there is a subsequence $\left\{u^{\varepsilon}, \varepsilon=\varepsilon_{k} \rightarrow 0\right\}$ that converges weakly in $W^{1, p_{0}(\cdot)}(\Omega)$ to a function $u$ such that the pair $\{u, A\}$ is a solution of

$$
J_{\text {hom }}[u, A] \longrightarrow \text { inf, } u \in W_{0}^{1, p_{0}(\cdot)}(\Omega) \text { with } A=\lim _{\varepsilon \rightarrow 0} A^{\varepsilon}
$$

Remark 10. It is important to notice that the constant $A$ in (45) remains unknown. Suppose, in addition, that the function $c(x, b)$ is differentiable with respect to the argument $b$. Then it is easy to see that Euler's equation for the homogenized problem (45) reads

$$
\begin{gathered}
-\operatorname{div}\left(|\nabla u|^{p_{0}(x)-2} \nabla u\right)+|u|^{p_{0}(x)-2} u+\mathbf{1}_{d}(x) c_{u}^{\prime}(x, u-A) \\
=f(x) \quad \text { in } \Omega ; \\
u=0 \quad \text { on } \partial \Omega, \quad \int_{\Omega_{d}} c_{u}^{\prime}(x, u-A) d x=0
\end{gathered}
$$

where $c_{u}^{\prime}$ denotes the partial derivative of the function $c$ with respect to $u$. This means that the homogenized problem (45) is nonlocal.

Theorem 9 is proved by arguments similar to those from the proof of Theorem 8 .

5.3. A Periodic Example. Theorems 8 and 9 provide sufficient conditions for the existence of the homogenized problem (45). The goal of this section is to prove that, for appropriate examples, all the conditions of Theorems 8 and 9 are satisfied and to compute the function $c(x, u)$ and the constant $A$ in the homogenized problem (45) explicitly.

Let $\Omega$ be a bounded Lipschitz domain in $\mathbb{R}^{3}$ and let $\Omega_{d}$ be the subdomain of $\Omega$ defined in condition (F.1.1). Let $\mathfrak{F}^{\varepsilon}$ be an 
$\varepsilon$-periodic coordinate lattice in $\mathbb{R}^{3}$ formed by the intersecting circular cylinders of radius $r_{\varepsilon}$,

$$
r_{\varepsilon}=e^{-1 / \varepsilon^{2}} \text {. }
$$

We set $\mathscr{F}^{\varepsilon}=\mathfrak{F}^{\varepsilon} \cap \Omega_{d}$ and $\Omega^{\varepsilon}=\Omega \backslash \overline{\mathscr{F}^{\varepsilon}}$.

Let $\left\{p_{\varepsilon}\right\}_{(\varepsilon>0)}$ be a class of continuous functions defined in the domain $\bar{\Omega}$. We assume that, for any $\varepsilon>0, p_{\varepsilon}$ satisfies the log-Hölder continuity condition and the following condition:

(B.1.2) the functions $p_{\varepsilon}$ are given by

$$
p_{\varepsilon}(x)= \begin{cases}2+\ell(x) \varepsilon^{2} & \text { in } \mathcal{N}\left(\mathscr{F}^{\varepsilon}, \varepsilon^{2}\right), \\ 2+\ell_{\varepsilon}(x) & \text { elsewhere }\end{cases}
$$

where $\mathcal{N}(\mathcal{O}, \delta)$ denotes the cylindrical $\delta$-neighborhood of the set $\mathcal{O}$ and where $\ell$ and $\ell_{\varepsilon}$ are smooth strictly positive functions in $\bar{\Omega}$, moreover, $\max _{x \in \bar{\Omega}} \ell_{\varepsilon}(x)=o(1)$ as $\varepsilon \rightarrow 0$.

Consider the boundary value problem

$$
\begin{gathered}
-\operatorname{div}\left(\left|\nabla u^{\varepsilon}\right|^{p_{\varepsilon}(x)-2} \nabla u^{\varepsilon}\right)+\left|u^{\varepsilon}\right|^{p_{\varepsilon}(x)-2} u^{\varepsilon}=f(x) \quad \text { in } \Omega^{\varepsilon}, \\
u^{\varepsilon}=A^{\varepsilon} \quad \text { on } \partial \mathscr{F}^{\varepsilon} ; \quad u^{\varepsilon}=0 \quad \text { on } \partial \Omega, \\
\int_{\partial \mathscr{F} \varepsilon}\left|\nabla u^{\varepsilon}\right|^{p_{\varepsilon}(x)-2} \frac{\partial u^{\varepsilon}}{\partial \vec{v}} d s=0,
\end{gathered}
$$

where $A^{\varepsilon}$ is an unknown constant. It is known from $[1,2]$ that, for each $\varepsilon>0$, there exists a unique solution $u^{\varepsilon} \in W^{1, p_{\varepsilon}(\cdot)}\left(\Omega^{\varepsilon}\right)$ of problem (49). We extend $u^{\varepsilon}$ by the equality $u^{\varepsilon}=A^{\varepsilon}$ in $\mathscr{F}^{\varepsilon}$ and keep for it the same notation. The asymptotic behavior of the solutions of problem (49) is given now by the following theorem.

Theorem 11. Let $u^{\varepsilon}$ be a solution of (49) extended by the equality $u^{\varepsilon}(x)=A^{\varepsilon}$ in $\mathscr{F}^{\varepsilon}$. Assume that the condition (B.1.2) on the function $p_{\varepsilon}$ is satisfied. Then the sequence $\left\{u^{\varepsilon}\right\}_{(\varepsilon>0)}$ converges weakly in $H^{1}(\Omega)$ to a function $u$ solution of

$$
\begin{gathered}
-\Delta u+u+\mu(x)\left(u-\mathrm{A}_{\ell}(u)\right)=g(x) \text { in } \Omega, \\
u=0 \quad \text { on } \partial \Omega,
\end{gathered}
$$

where

$$
\begin{aligned}
& \mu(x)=6 \pi \mathbf{1}_{d}(x) \frac{e^{\ell(x)}-1}{\ell(x)} \\
& \mathrm{A}_{\ell}(u)=\left(\int_{\Omega_{d}} \mu(x) d x\right)^{-1} \int_{\Omega_{d}} \mu(x) u(x) d x .
\end{aligned}
$$

Theorem 11 is proved in [23] and is based essentially on the calculation of the local energy characteristic for this periodic structure.

Remark 12. It is easy to see from the proof of Theorem 11 that if in (48) we replace $\ell(x) \varepsilon^{2}$ by a function $\tilde{\ell}_{\varepsilon}$ with $\max _{x \in \bar{\Omega}} \tilde{\ell}_{\varepsilon}=$ $o\left(\varepsilon^{2}\right)$, then $\mu(x)=6 \pi \mathbf{1}_{d}(x)$ as in the linear case.
Remark 13. It is shown in [27] Paragraph 3.3 that for the integrands of growth $\left|\nabla u^{\varepsilon}\right|^{2} \ln \left|\nabla u^{\varepsilon}\right|$, the 3D lattice becomes extremely thin. Moreover, for the integrands of growth $\left|\nabla u^{\varepsilon}\right|^{2+\delta}$, where $\delta>0$ is a parameter independent of $\varepsilon$, there is no $3 \mathrm{D}$ lattice which admits nontrivial homogenization result, because the capacity of the lattice goes to infinity as $\varepsilon \rightarrow 0$. Theorem 11 shows the maximal possible polynomial growth of the integrand (in a small neighborhood of the lattice) which admits a nontrivial homogenization result.

\section{Homogenization of the Neumann Problem}

6.1. Statement of the Problem and Main Results. Let $\Omega$ be a bounded Lipschitz domain in $\mathbb{R}^{n}(n \geq 2)$. Let $\left\{\mathscr{F}^{\varepsilon}\right\}_{(\varepsilon>0)}$ be a family of open subsets in $\Omega$; in the sequel $\varepsilon$ is a small positive parameter characterizing the microscopic length scale. We assume that the set $\mathscr{F}^{\varepsilon}$ consists of $N_{\varepsilon}\left(N_{\varepsilon} \rightarrow+\infty\right.$ as $\left.\varepsilon \rightarrow 0\right)$ small isolated components such that their diameters go to zero as $\varepsilon \rightarrow 0$ and $\mathscr{F}^{\varepsilon}$ is distributed in an asymptotically regular way in $\Omega$. We set

$$
\Omega^{\varepsilon}=\Omega \backslash \overline{\mathscr{F}^{\varepsilon}} .
$$

We assume that a family of continuous functions $\left\{p_{\varepsilon}\right\}_{(\varepsilon>0)}$ belongs to the class $\mathfrak{Q}_{p_{0}(\cdot)}^{\varepsilon}$. On the space $L^{p_{\varepsilon}(\cdot)}\left(\Omega^{\varepsilon}\right)$, we define the functional $J^{\varepsilon}: L^{p_{\varepsilon}(\cdot)}\left(\Omega^{\varepsilon}\right) \rightarrow \mathbb{R} \cup\{+\infty\}:$

$$
J^{\varepsilon}[u]= \begin{cases}\int_{\Omega^{\varepsilon}} \mathrm{F}_{\varepsilon}(x, u, \nabla u) d x, & \text { if } u \in W^{1, p_{\varepsilon}(\cdot)}\left(\Omega^{\varepsilon}\right) \\ +\infty, & \text { otherwise }\end{cases}
$$

where $\mathrm{F}_{\varepsilon}(x, u, \nabla u)$ is defined in (31) and $f \in C(\bar{\Omega})$.

We study the asymptotic behavior of $J^{\varepsilon}$ and their minimizers as $\varepsilon \rightarrow 0$. The classical periodicity assumption is here substituted by an abstract one covering a variety of concrete behaviors, such as the periodicity, the almost periodicity, and many more besides. For this, we assume that $\Omega^{\varepsilon} \subset \Omega$ is a disperse medium; that is, the following assumptions hold:

(C.2.1) the local concentration of the set $\Omega^{\varepsilon}$ has a positive continuous limit, that is, the indicator of $\Omega^{\varepsilon}$ converges weakly in $L^{2}(\Omega)$ to a continuous positive limit. This implies that there exists a continuous positive function $\rho=\rho(x)$ such that

$$
\lim _{h \rightarrow 0} \lim _{\varepsilon \rightarrow 0} h^{-n} \operatorname{meas}\left(K_{h}^{x} \cap \Omega^{\varepsilon}\right)=\rho(x),
$$

for any open cube $K_{h}^{x}$ centered at $x \in \Omega$ with lengths equal to $h>0$;

(C.2.2) for any $q \in\left[p^{-}, p^{+}\right]$, there exists a family of extension operators $\mathrm{P}_{q}^{\varepsilon}: W^{1, q}\left(\Omega^{\varepsilon}\right) \rightarrow W^{1, q}(\Omega)$ such that.

global: for any $u^{\varepsilon} \in W^{1, q}\left(\Omega^{\varepsilon}\right)$,

$$
\begin{aligned}
& \left\|\mathrm{u}^{\mathcal{\varepsilon}}\right\|_{L^{q}(\Omega)} \leq C\left\|u^{\varepsilon}\right\|_{L^{q}\left(\Omega^{\varepsilon}\right)}, \\
& \left\|\nabla \mathbf{u}^{\varepsilon}\right\|_{L^{q}(\Omega)} \leq C\left\|\nabla u^{\varepsilon}\right\|_{L^{q}\left(\Omega^{\varepsilon}\right)},
\end{aligned}
$$




$$
\begin{aligned}
& \text { uniformly in } \varepsilon>0 \text {, where } \mathrm{u}^{\varepsilon}=\mathrm{P}^{\varepsilon} u^{\varepsilon} \text { and } \mathrm{u}^{\varepsilon}=u^{\varepsilon} \text { in } \\
& \Omega^{\varepsilon} ; \\
& \text { local: for any } h>0 \text { there is } \varepsilon_{0}(h)>0 \text { such that for } \\
& \text { all } \varepsilon<\varepsilon_{0}(h), z \in \Omega \text { and any function } u \in W^{1, q}((z+ \\
& \left.\left.[-2 h, 2 h]^{n}\right) \cap \Omega^{\varepsilon}\right) \text {, the estimates hold } \\
& \left\|u^{\varepsilon}\right\|_{L^{q}\left(\left(z+[-h, h]^{n}\right) \cap \Omega\right)} \leq C\left\|u^{\varepsilon}\right\|_{L^{q}\left(\left(z+[-2 h, 2 h]^{n}\right) \cap \Omega^{\varepsilon}\right)^{\prime}} \\
& \left\|\nabla u^{\varepsilon}\right\|_{L^{q}\left(\left(z+[-h, h]^{n}\right) \cap \Omega\right)} \leq C\left\|\nabla u^{\varepsilon}\right\|_{L^{q}\left(\left(z+[-2 h, 2 h]^{n}\right) \cap \Omega^{\varepsilon}\right)^{*}}
\end{aligned}
$$

Remark 14. Notice that in condition (C.2.2), we require the existence of extension operators only in usual Sobolev spaces $W^{1, q}$ with constant $q$. In this case, the extension condition is well studied in the mathematical literature (see, e.g., [27, 30$32])$. For instance, it holds for a wide class of disperse media (see, for instance, [27]).

One more condition is imposed on the local characteristic of the set $\mathscr{F}^{\varepsilon}$ associated to the functional (53). In order to formulate this condition, we denote by $K_{h}^{z}$ an open cube centered at $z \in \Omega$ with edge length $h(0<\varepsilon \ll h \ll 1)$ and introduce the functional

$$
\begin{aligned}
c_{p_{\varepsilon}(\cdot)}^{\varepsilon, h}(z, \mathbf{b})=\inf _{v^{\varepsilon}} \int_{K_{h}^{z} \cap \Omega^{\varepsilon}}\{ & \frac{1}{p_{\varepsilon}(x)}\left|\nabla v^{\varepsilon}\right|^{p_{\varepsilon}(x)} \\
& \left.+h^{-p_{\varepsilon}(x)-\gamma}\left|v^{\varepsilon}-(x-z, \mathbf{b})\right|^{p_{\varepsilon}(x)}\right\} d x,
\end{aligned}
$$

where $\gamma>0, \mathbf{b} \in \mathbb{R}^{n}$, and the infimum is taken over $v^{\varepsilon} \in$ $W^{1, p_{\varepsilon}(\cdot)}\left(K_{h}^{z} \cap \Omega^{\varepsilon}\right)$. Here $(\cdot, \cdot)$ stands for the scalar product in $\mathbb{R}^{n}$. We assume that

(C.2.3) there is a continuous, with respect to $x \in \bar{\Omega}$, function $T(x, \mathbf{b})$ and $\gamma=\gamma_{0}\left(0<\gamma_{0}<p^{-}\right)$such that for any $\left\{p_{\varepsilon}\right\}_{(\varepsilon>0)} \subset \mathfrak{Q}_{p_{0}(\cdot)}^{\varepsilon}$, any $x \in \Omega$ and any $\mathbf{b} \in \mathbb{R}^{n}$,

$\lim _{h \rightarrow 0} \varlimsup_{\varepsilon \rightarrow 0} h^{-n} c_{p_{\varepsilon}(\cdot)}^{\varepsilon, h}(x, \mathbf{b})=\lim _{h \rightarrow 0} \frac{\lim _{\varepsilon \rightarrow 0}}{h^{-n}} c_{p_{\varepsilon}(\cdot)}^{\varepsilon, h}(x, \mathbf{b})=T(x, \mathbf{b})$.

Remark 15. Condition (C.2.3) is always fulfilled for periodic and locally periodic structures.

Remark 16. It is crucial in condition (C.2.3) that the limit function $T(x, \mathbf{b})$ does not depend on the particular choice of the sequence $p_{\varepsilon} \rightarrow p_{0}$. Notice that this is always the case for periodic and locally periodic perforated media. These media will be considered in detail in the last section of the paper.

Now we are in position to formulate the first convergence result of the section.

Theorem 17. Assume that $\left\{p_{\varepsilon}\right\}_{(\varepsilon>0)} \subset{\mathfrak{\mathfrak { R } ^ { \varepsilon }}}_{p_{0}(\cdot)}$, and let conditions (C.2.1)-(C.2.3) be satisfied. Then the functionals $J^{\varepsilon}$ defined in

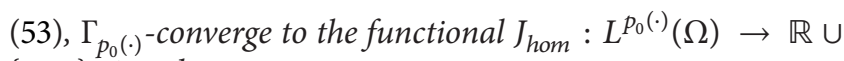
$\{+\infty\}$ given by

$$
\begin{aligned}
& J_{\text {hom }}[u] \\
& =\left\{\begin{array}{cl}
\int_{\Omega}\left\{T(x, \nabla u)+\frac{\rho(x)}{p_{0}(x)}|u|^{p_{0}(x)}\right. & \\
-\rho(x) f(x) u\} d x, & \text { if } u \in W^{1, p_{0}(\cdot)}(\Omega), \\
+\infty, & \text { otherwise. }
\end{array}\right.
\end{aligned}
$$

Now let us formulate the convergence result for the minimizers of the functionals $J^{\varepsilon}$. Consider the variational problem

$$
J^{\varepsilon}\left[u^{\varepsilon}\right] \longrightarrow \min , \quad u^{\varepsilon} \in W^{1, p_{\varepsilon}(\cdot)}\left(\Omega^{\varepsilon}\right)
$$

According to $[1-3,6]$, for each $\varepsilon>0$, problem (60) has a unique solution $u^{\varepsilon} \in W^{1, p_{\varepsilon}(\cdot)}\left(\Omega^{\varepsilon}\right)$.

The following convergence result holds.

Theorem 18. Under the assumptions of Theorem 17, the solution $u^{\varepsilon}$ of the variational problem (60) converges strongly in $L^{p_{0}(\cdot)}\left(\Omega^{\varepsilon}\right)$ to a solution of the problem

$$
J_{\text {hom }}[u] \longrightarrow \min , \quad u \in W^{1, p_{0}(\cdot)}(\Omega) .
$$

The Scheme of the Proof of Theorems 17 and 18 (See [22] for More Details) Is as Follows. The "lim inf"-inequality is proved in two steps as in Theorem 7 by introducing an auxiliary functional. The "lim sup"-inequality is proved by the arguments similar to those from the proof of Theorem 8 .

6.2. A Periodic Example. Theorems 17 and 18 of Section 6.1 provide sufficient conditions for the existence of the $\Gamma$ limit functional (59) and for the convergence of minimizers of the variational problem (60) to the minimizer of the homogenized variational problem (61). It is important to show that the class of functions which satisfy the conditions of these theorems is not empty. The goal of this section is to prove that for periodic and locally periodic media all conditions of the above-mentioned theorems are satisfied and to compute the coefficients of the homogenized functional (59) in terms of solutions of auxiliary cell problems. In fact, conditions (C.2.1) and (C.2.3) are always satisfied in the periodic case if the boundary of inclusions is regular enough, and that the extension condition (C.2.2) can also be replaced with the assumption on the regularity of the inclusions geometry.

Let $\Omega$ be a bounded domain in $\mathbb{R}^{n}(n \geq 2)$ with sufficiently smooth boundary. We assume that in the periodic cell $Y=] 0,1{ }^{n}$, there is an obstacle $F \Subset Y$ being an open set with a sufficiently smooth boundary $\partial F$. We assume that this geometry is repeated periodically in the whole $\mathbb{R}^{n}$. The geometric structure within the domain $\Omega$ is then obtained by intersecting the $\varepsilon$-multiple of this geometry with $\Omega$. Let $\left\{x^{\mathbf{k}, \varepsilon}\right\}$ be an $\varepsilon$-periodic grid in $\mathbb{R}^{n}: x^{\mathbf{k}, \varepsilon}=\varepsilon \mathbf{k}, \mathbf{k} \in \mathbb{Z}^{n}$. Then we 
define $\mathscr{F}^{\varepsilon}$ as the union of sets $\mathscr{F}_{\mathbf{k}}^{\varepsilon} \subset K_{\varepsilon}^{\mathbf{k}}$ obtained from $\varepsilon F$ by translations with vectors $\varepsilon \mathbf{k}, \mathbf{k} \in \mathbb{Z}^{n}$, that is,

$$
\mathscr{F}_{\mathbf{k}}^{\varepsilon}=\varepsilon \mathbf{k}+\varepsilon F, \quad \mathscr{F}^{\varepsilon}=\bigcup_{\mathbf{k}}\left(\mathscr{F}_{\mathbf{k}}^{\varepsilon} \cap \Omega\right),
$$

and $K_{\varepsilon}^{\mathbf{k}}=\varepsilon \mathbf{k}+\varepsilon Y$. Notice that the geometry of the inclusions having a nontrivial intersection with the domain boundary might be rather complicated. In particular, the extension condition (C.2.2) might be violated for these inclusions. To avoid these technical difficulties, we assume that the domain $\Omega$ is not perforated in a small neighborhood of its boundary $\partial \Omega$. We set

$$
\Omega^{\varepsilon} \stackrel{\text { def }}{=} \Omega \backslash \overline{\mathscr{F}}^{\varepsilon} .
$$

Let a family of continuous functions $\left\{p_{\varepsilon}\right\}_{(\varepsilon>0)}$ belongs to the class ${\mathfrak{Q ^ { 2 }}}_{p_{0}(\cdot)}$. On the space $L^{p_{\varepsilon}(\cdot)}\left(\Omega^{\varepsilon}\right)$, we define the functional $J^{\varepsilon}: L^{p_{\varepsilon}(\cdot)}\left(\Omega^{\varepsilon}\right) \rightarrow \mathbb{R} \cup\{+\infty\}:$

$$
\begin{aligned}
& J^{\varepsilon}[u] \\
& =\left\{\begin{array}{cl}
\int_{\Omega^{\varepsilon}}\left\{\frac{1}{p_{\varepsilon}(x)}\left(|\nabla u|^{p_{\varepsilon}(x)}+|u|^{p_{\varepsilon}(x)}\right)\right. & \\
-f(x) u\} d x, & \text { if } u \in W^{1, p_{\varepsilon}(\cdot)}\left(\Omega^{\varepsilon}\right), \\
+\infty, & \text { otherwise, }
\end{array}\right.
\end{aligned}
$$

where $f \in C(\bar{\Omega})$.

We study the asymptotic behavior of the functional $J^{\varepsilon}$ and its minimizer as $\varepsilon \rightarrow 0$. To formulate the main result of this section we will introduce some notation. We denote by $U^{\mathbf{b}}=$ $U^{\mathbf{b}}(p, y)$ a minimizer of the following variational problem:

$$
\int_{Y^{\star}} \frac{1}{p}\left|\nabla_{y} U^{\mathbf{b}}-\mathbf{b}\right|^{p} d y \longrightarrow \min , \quad u \in W_{\mathrm{per}}^{1, p}\left(Y^{\star}\right),
$$

where $Y^{\star}=Y \backslash \bar{F}$, and $\mathbf{b}=\left(b_{1}, b_{2}, \ldots, b_{n}\right)$ is a vector in $\mathbb{R}^{n}$, and $p>1$ is a parameter.

If $p \geq 2$, then the solution $U^{\mathbf{b}}$ coincides with a unique solution in $W_{\text {per }}^{1, p}\left(Y^{\star}\right)$ of the following cell problem:

$$
\begin{gathered}
\operatorname{div}_{y}\left(\left|\nabla_{y} U^{\mathbf{b}}\right|^{p-2} \nabla_{y} U^{\mathbf{b}}\right)=0 \quad \text { in } Y^{\star}, \\
\left(\left|\nabla_{y} U^{\mathbf{b}}\right|^{p-2} \nabla_{y} U^{\mathbf{b}}-\mathbf{b}, \vec{\nu}\right)=0 \quad \text { on } \partial F, \\
y \longrightarrow U^{\mathbf{b}}(y) \quad Y \text {-periodic, }
\end{gathered}
$$

here $\vec{\nu}$ is the outward normal to $\partial F$.

The following result holds.
Theorem 19. The sequence of functionals $\left\{J^{\varepsilon}\right\}_{(\varepsilon>0)}$ defined in (64) $\Gamma_{p_{0}(\cdot)}$-converges to the functional $J_{\text {hom }}: L^{p_{0}(\cdot)}(\Omega) \rightarrow \mathbb{R} \cup$ $\{+\infty\}$ given by

$$
\begin{aligned}
& J_{\text {hom }}[u] \\
& =\left\{\begin{array}{cl}
\int_{\Omega}\left\{T(x, \nabla u)+\frac{\rho}{p_{0}(x)}|u|^{p_{0}(x)}\right. & \\
-\rho f(x) u\} d x, & \text { if } u \in W^{1, p_{0}(\cdot)}(\Omega), \\
+\infty, & \text { otherwise, }
\end{array}\right.
\end{aligned}
$$

where

$$
\begin{gathered}
\rho=\text { meas } Y^{\star} \\
T(x, \mathbf{b})=\int_{Y^{\star}} \frac{1}{p_{0}(x)}\left|\nabla_{y} U^{\mathbf{b}}\left(p_{0}(x), y\right)-\mathbf{b}\right|^{p_{0}(x)} d y .
\end{gathered}
$$

Moreover, a minimizer $u^{\varepsilon}$ of the functional (64) converges strongly in the space $L^{p_{0}(\cdot)}\left(\Omega^{\varepsilon}\right)$ to $u$ the minimizer of the homogenized functional (67).

Notice that Theorem 19 (see [22]) can be proved in two different ways. One of them is to check that under the assumptions of Theorem 19 conditions (C.2.1)-(C.2.3) are satisfied and that the characteristics introduced in conditions (C.2.1) and (C.2.3) coincide with those defined in (68). On the other hand, in the periodic case, the direct $\Gamma$-convergence techniques can be applied. This allows us to obtain formula (68) by means of $\Gamma$-convergence approach used in periodic homogenization.

\section{Homogenization of Quasilinear Elliptic Equations with Nonstandard Growth in High-Contrast Media}

7.1. Statement of the Problem and the Main Result. Let $\Omega=$ $\Omega_{f}^{\varepsilon} \cup \overline{\Omega_{m}^{\varepsilon}}$ be a bounded domain of $\mathbb{R}^{n}(n \geq 2)$ with Lipschitz boundary $\partial \Omega$. Here $\left\{\Omega_{m}^{\varepsilon}\right\}_{(\varepsilon>0)}$ is a family of open subsets in $\Omega$. We assume that the set $\Omega_{m}^{\varepsilon}$ is distributed in an asymptotically regular way in $\Omega$; moreover, for the sake of simplicity, we suppose that $\Omega_{m}^{\varepsilon} \cap \partial \Omega=\emptyset$.

Remark 20. In the framework of the method of local energy characteristics presented in the section, we do not specify the geometrical structure of the set $\Omega_{m}^{\varepsilon}$. Generally speaking, it may consist of $N_{\varepsilon}\left(N_{\varepsilon} \rightarrow+\infty\right.$ as $\left.\varepsilon \rightarrow 0\right)$ small isolated components such that their diameters go to zero as $\varepsilon \rightarrow 0$ or it may be defined as fibres becoming more and more dense as $\varepsilon \rightarrow 0$, such that the diameters of the fibers go to zero as $\varepsilon \rightarrow 0$.

We assume that the family of functions $\left\{p_{\varepsilon}\right\}_{(\varepsilon>0)}$ belongs to the class $\mathfrak{\Omega}_{p_{0}(\cdot)}^{\varepsilon}$. Let $\sigma \in C(\bar{\Omega})$ be such that 
(A.3.4) there exist two real numbers $\sigma^{-}$and $\sigma^{+}$such that the function $\sigma$ is bounded in the following sense:

$$
\begin{aligned}
0<\sigma^{-} & \equiv \min _{x \in \bar{\Omega}} \sigma(x) \leq \sigma(x) \leq \max _{x \in \bar{\Omega}} \sigma(x) \\
& \equiv \sigma^{+}<\min _{x \in \bar{\Omega}} \frac{p_{0}(x) n}{n-p_{0}(x)} \quad \text { in } \bar{\Omega}
\end{aligned}
$$

(A.3.5) the function $\sigma$ satisfies the log-Hölder continuity property.

Let us now define the variational problem under consideration. To this end, we consider the functional $J^{\varepsilon}$ : $W^{1, p_{\varepsilon}(\cdot)}(\Omega) \rightarrow \mathbb{R} \cup\{+\infty\}$,

$$
J^{\varepsilon}[u] \stackrel{\operatorname{def}}{=} \begin{cases}\int_{\Omega} \mathrm{G}_{\varepsilon}(x, u, \nabla u) d x & \text { if } u \in W^{1, p_{\varepsilon}(\cdot)}(\Omega), \\ +\infty & \text { otherwise, }\end{cases}
$$

where

$$
\begin{aligned}
\mathrm{G}_{\varepsilon}(x, u, \nabla u) \stackrel{\text { def }}{=} & \varkappa_{\varepsilon}(x)|\nabla u|^{p_{\varepsilon}(x)}+\frac{1}{\sigma(x)}|u|^{\sigma(x)} \\
& -g^{\varepsilon}(x) u \quad \text { with } \varkappa_{\varepsilon}(x) \stackrel{\text { def }}{=} \frac{K_{\varepsilon}(x)}{p_{\varepsilon}(x)} .
\end{aligned}
$$

Here the function $g^{\varepsilon}$ is defined by $g^{\varepsilon}(x) \stackrel{\text { def }}{=} \mathbf{1}_{f}^{\varepsilon}(x) g(x), g \in$ $C(\Omega)$. We denote by $\mathbf{1}_{k}^{\varepsilon}$ the characteristic function of the set $\Omega_{k}^{\varepsilon}, k=f, m . K_{\varepsilon}$ is a measurable function in $\Omega$ such that

(K.1) there exists a real number $k_{0}$ such that $0<k_{0} \leq$ $K_{\varepsilon}(x) \leq k_{0}^{-1}$ in $\Omega_{f}^{\varepsilon}$;

(K.2) for any $\varepsilon>0$, there exists a real number $\mathrm{k}_{\varepsilon}$ such that $\sup _{x \in \Omega_{m}^{\varepsilon}} K_{\varepsilon}(x)=\mathrm{k}_{\varepsilon}>0$ and $\mathrm{k}_{\varepsilon} \rightarrow 0$ as $\varepsilon \rightarrow 0$.

We consider the following variational problem:

$$
J^{\varepsilon}\left[u^{\varepsilon}\right] \longrightarrow \min , \quad u^{\varepsilon} \in W_{0}^{1, p_{\varepsilon}(\cdot)}(\Omega) .
$$

It is known from [1] that for each $\varepsilon>0$, there exists a unique solution $u^{\varepsilon} \in W_{0}^{1, p_{\varepsilon}(\cdot)}(\Omega)$ of the variational problem (72). We aim to study the asymptotic behavior of the family $\left\{u^{\varepsilon}\right\}$ as $\varepsilon \rightarrow 0$, bearing in mind that the geometry of $\Omega=\Omega_{f}^{\varepsilon} \cup \bar{\Omega}_{m}^{\varepsilon}$ depends on $\varepsilon$. So, we have to specify this geometry. Most of the papers dealing with homogenization assume that $\Omega$ is a periodic repetition of a standard cell. This classical periodicity assumption is here substituted by an abstract one covering a variety of concrete behaviors including periodicity and almost periodicity. We thus make the following assumptions:

(C.3.1) the local concentration of the set $\Omega_{f}^{\varepsilon}$ has a positive continuous limit; that is, the indicator of $\Omega_{f}^{\varepsilon}$ converges weakly in $L^{2}(\Omega)$ to a continuous positive limit. This implies that there exists a continuous positive function $\rho=\rho(x)$ such that

$$
\lim _{h \rightarrow 0} \lim _{\varepsilon \rightarrow 0} h^{-n} \operatorname{meas}\left(K_{h}^{x} \cap \Omega_{f}^{\varepsilon}\right)=\rho(x)
$$

for any open cube $K_{h}^{x}$ centered at $x \in \Omega$ with lengths equal to $h>0$;

(C.3.2) for any $\left\{p_{\varepsilon}\right\}_{(\varepsilon>0)} \subset \mathfrak{Q}_{p_{0}(\cdot)}^{\varepsilon}$, there is a constant $C_{p_{\varepsilon}} \geq 0$ such that if the function $p_{\varepsilon}^{\star}$ is defined by $p_{\varepsilon}^{\star}(x)=$ $p_{\varepsilon}(x)-C_{p_{\varepsilon}}$ in $\Omega$, then:

(i) the sequence $\left\{p_{\varepsilon}^{\star}\right\}_{(\varepsilon>0)}$ belongs to $\mathfrak{R}_{p_{0}(\cdot)}^{\varepsilon}$, that is, $\lim _{\varepsilon \rightarrow 0} C_{p_{\varepsilon}}=0$;

(ii) there exists a family of extension operators $\mathbf{P}^{\varepsilon}$ : $W^{1, p_{\varepsilon}^{\star}(\cdot)}\left(\Omega_{f}^{\varepsilon}\right) \rightarrow W^{1, p_{\varepsilon}^{\star}(\cdot)}(\Omega)$ such that, for any $v^{\varepsilon} \in$ $W^{1, p_{\varepsilon}(\cdot)}\left(\Omega_{f}^{\varepsilon}\right)$,

$$
\begin{gathered}
\mathbf{P}^{\varepsilon} v^{\varepsilon}=v^{\varepsilon} \quad \text { in } \Omega_{f}^{\varepsilon}, \\
\left\|\mathbf{P}^{\varepsilon} v^{\varepsilon}\right\|_{W^{1, p_{\varepsilon}^{\star}(\cdot)}(\Omega)} \leq \Phi\left(\left\|v^{\varepsilon}\right\|_{W^{1, p_{\varepsilon}(\cdot)}\left(\Omega_{f}^{\varepsilon}\right)}\right),
\end{gathered}
$$

where $\Phi=\Phi(t)$ is a strictly monotone continuous function in $\mathbb{R}^{+}$such that $\Phi(0)=0$ and $\Phi(t) \rightarrow+\infty$ as $t \rightarrow+\infty$.

We also impose several conditions on the local characteristic of the set $\Omega_{f}^{\varepsilon}$ and $\Omega_{m}^{\varepsilon}$ associated to the functional (70). Let $K_{h}^{z}$ be an open cube centered at $z \in \Omega$ with lengths equal to $h(0<\varepsilon \ll h \ll 1)$. We introduce the following functionals:

(i) the functional $c_{p_{\varepsilon}(\cdot)}^{\varepsilon, h}$ associated to the energy in $\Omega_{f}^{\varepsilon}$ is defined in $\Omega \times \mathbb{R}^{n}$ by

$$
\begin{aligned}
& c_{p_{\varepsilon}(\cdot)}^{\varepsilon, h}(z ; \mathbf{a}) \\
& \stackrel{\text { def }}{=} \inf _{v^{\varepsilon}} \int_{K_{h}^{z} \cap \Omega_{f}^{\varepsilon}}\left\{\varkappa_{\varepsilon}(x)\left|\nabla v^{\varepsilon}(x)\right|^{p_{\varepsilon}(x)}\right. \\
& \left.+h^{-p_{\varepsilon}(x)-\gamma}\left|v^{\varepsilon}(x)-(x-z, \mathbf{a})\right|^{p_{\varepsilon}(x)}\right\} d x,
\end{aligned}
$$

for $z \in \Omega$, a $\in \mathbb{R}^{n}$, where $\gamma>0$ and the infimum is taken over $v^{\varepsilon} \in W^{1, p_{\varepsilon}(\cdot)}\left(K_{h}^{z} \cap \Omega_{f}^{\varepsilon}\right)$.

(ii) The functional $b_{p_{\varepsilon}(\cdot)}^{\varepsilon, h}$ associated to the energy exchange between $\Omega_{f}^{\varepsilon}$ and $\Omega_{m}^{\varepsilon}$ is defined in $\Omega \times \mathbb{R}^{n}$ by

$$
\begin{aligned}
& b_{p_{\varepsilon}(\cdot)}^{\varepsilon, h}(z ; \beta) \\
& \stackrel{\text { def }}{=} \inf _{w^{\varepsilon}} \int_{K_{h}^{z}}\left\{\varkappa_{\varepsilon}(x)\left|\nabla w^{\varepsilon}\right|^{p_{\varepsilon}(x)}+\frac{\mathbf{1}_{m}^{\varepsilon}(x)}{\sigma(x)}\left|w^{\varepsilon}\right|^{\sigma(x)}\right. \\
& \left.+h^{-p_{\varepsilon}(x)-\gamma} \mathbf{1}_{f}^{\varepsilon}(x)\left|w^{\varepsilon}-\beta\right|^{p_{\varepsilon}(x)}\right\} d x,
\end{aligned}
$$

for $z \in \Omega, \beta \in \mathbb{R}$, the infimum being taken over $w^{\varepsilon} \in$ $W^{1, p_{\varepsilon}(\cdot)}\left(K_{h}^{z}\right)$

We assume that the local characteristics of $\Omega$ are such that 
(C.3.3) for any $x \in \Omega$ and any $\mathbf{a} \in \mathbb{R}^{n}$, there is a continuous function $T(x, \mathbf{a})$ and a real number $\gamma=\gamma_{0}\left(0<\gamma_{0}<\right.$ $\left.p^{-}\right)$such that, for any $\left\{p_{\varepsilon}\right\}_{(\varepsilon>0)} \subset \mathfrak{Q}_{p_{0}(\cdot)}^{\varepsilon}$,

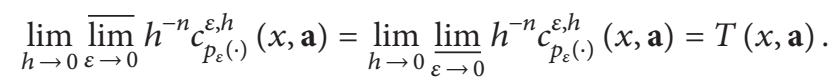

(C.3.4) for any $x \in \Omega$ and any $\beta \in \mathbb{R}$, there is a continuous function $\mathrm{b}(x, \beta)$ and a real number $\gamma=\gamma_{1}\left(0<\gamma_{1}<\right.$ $\left.p^{-}\right)$such that, for any $\left\{p_{\varepsilon}\right\}_{(\varepsilon>0)} \subset \mathfrak{Q}_{p_{0}(\cdot)}^{\varepsilon}$,

$\lim _{h \rightarrow 0} \varlimsup_{\varepsilon \rightarrow 0} h^{-n} b_{p_{\varepsilon}(\cdot)}^{\varepsilon, h}(x, \beta)=\lim _{h \rightarrow 0} \lim _{\varepsilon \rightarrow 0} h^{-n} b_{p_{\varepsilon}(\cdot)}^{\varepsilon, h}(x, \beta)=\mathrm{b}(x, \beta)$.

Remark 21. It is crucial in conditions (C.3.3) and (C.3.4) that the limit functions $T(x, \mathbf{b})$ and $b(x, \beta)$ do not depend on the particular choice of the sequence $\left\{p_{\varepsilon}\right\}_{(\varepsilon>0)} \subset \mathfrak{\Omega}_{p_{0}(\cdot)}^{\varepsilon}$. It is proved in [26] that these assumptions are fulfilled for periodic and locally periodic media.

Remark 22. Contrary to the standard growth setting as considered in $[20,32]$, the local characteristic $b_{p_{\varepsilon}(\cdot)}^{\varepsilon, h}(z ; \beta)$ is not homogeneous with respect to the parameter $\beta$. This induces the appearance of a nonlinear function $\mathrm{b}(x, u)$ in the homogenized functional (see Theorem 23 below).

The main result of the section is the following.

Theorem 23. Let $u^{\varepsilon}$ be a solution of (72). Assume that $p^{\varepsilon} \in \mathfrak{Q}_{p_{0}(\cdot)}^{\varepsilon}$ and conditions (A.3.4)-(A.3.5), (K.1)-(K.2), and (C.3.1)-(C.3.4) are satisfied. Then $u^{\varepsilon}$, solution of the variational problem (72), converges strongly in $L^{p_{0}(\cdot)}\left(\Omega_{f}^{\varepsilon}\right)$ to $u$, solution of the following variational problem:

$$
J_{\text {hom }}[u] \longrightarrow \min , \quad u \in W_{0}^{1, p_{0}(\cdot)}(\Omega),
$$

the homogenized functional $J_{\text {hom }}: W_{0}^{1, p_{0}(\cdot)}(\Omega) \rightarrow \mathbb{R} \cup\{+\infty\}$ being defined by

$$
J_{\text {hom }}[u] \stackrel{\text { def }}{=} \begin{cases}\int_{\Omega} G_{0}(x, u, \nabla u) d x & \text { if } u \in W_{0}^{1, p_{0}(\cdot)}(\Omega), \\ +\infty & \text { otherwise, }\end{cases}
$$

where

$$
\begin{aligned}
\mathrm{G}_{0}(x, u, \nabla u) \stackrel{\text { def }}{=} & T(x, \nabla u)+\frac{\rho(x)}{\sigma(x)}|u|^{\sigma(x)} \\
& +\mathrm{b}(x, u)-g(x) \rho(x) u .
\end{aligned}
$$

Moreover, for any smooth function $\zeta$ in $\Omega$, we have

$$
\begin{aligned}
\lim _{\varepsilon \rightarrow 0} \int_{\Omega_{m}^{\varepsilon}}\left\{\frac{1}{p_{\varepsilon}(x)}\left|u^{\varepsilon}\right|^{\sigma(x)-2}\left(u(x) u^{\varepsilon}-\left|u^{\varepsilon}\right|^{2}\right)\right. \\
\left.\quad+\frac{1}{\sigma(x)}\left|u^{\varepsilon}\right|^{\sigma(x)}\right\} \zeta(x) d x=\int_{\Omega} \mathrm{b}(x, u) \zeta(x) d x .
\end{aligned}
$$

The scheme of the proof of Theorem 23 (see [26] for more details) is similar to the scheme of the proof of Theorems 17 and 18.

7.2. Periodic Examples. Theorem 23 of Section 7.1 provides sufficient conditions for the existence of the homogenized functional (80) and for the convergence of minimizers of the variational problem (72) to the minimizer of the homogenized variational problem (79) under conditions (A.3.4)(A.3.5), (K.1)-(K.2), and (C.3.1)-(C.3.4). It is important to show that the "intersection" of these conditions is not empty. The goal of this section is to prove that for periodic media all the conditions of the above-mentioned theorem are satisfied and to compute the coefficients of the homogenized functional (80) either in an explicit form or as usually by the solution of a corresponding cell problem.

Let $\Omega$ be a bounded domain in $\mathbb{R}^{n}(n \geq 2)$ with Lipschitz boundary. We assume that in the standard periodic cell $Y \stackrel{\text { def }}{=}$ ] $0,1\left[{ }^{n}\right.$, there is an obstacle $M \subset Y$ with Lipschitz boundary $\partial M$. We assume that this geometry is repeated periodically in the whole $\mathbb{R}^{n}$. The geometric structure within the domain $\Omega$ is then obtained by intersecting the $\varepsilon$-multiple of this geometry with $\Omega$. Let $\left\{x^{i, \varepsilon}\right\}$ be an $\varepsilon$-periodic grid in $\Omega$. Then we define $\Omega_{m}^{\varepsilon}$ as the union of sets $M_{i}^{\varepsilon} \subset K_{\varepsilon}^{i}\left(i=1,2, \ldots, N_{\varepsilon}\right)$ obtained from $\varepsilon M$ by translations of vectors $\varepsilon \sum_{j=1}^{n} k_{j} \mathbf{e}_{j}$, that is,

$$
\Omega_{m}^{\varepsilon}=\bigcup_{i}^{N_{\varepsilon}} M_{i}^{\varepsilon}, \quad \Omega_{f}^{\varepsilon}=\Omega \backslash \overline{\Omega_{m}^{\varepsilon}},
$$

where $K_{\varepsilon}^{i}$ is the cube centered at the point $x^{i, \varepsilon}$ and of length $\varepsilon, k_{j} \in \mathbb{Z},\left\{\mathbf{e}_{j}\right\}_{j=1}^{n}$ is the canonical basis of $\mathbb{R}^{n}$, and $N_{\varepsilon} \rightarrow+\infty$ as $\varepsilon \rightarrow 0$. that

Let $p_{0}=p_{0}(x)$ be a log-Hölder continuous function such

$$
\begin{aligned}
2<p^{-} & \equiv \min _{x \in \bar{\Omega}} p_{0}(x) \leq p_{0}(x) \leq \max _{x \in \bar{\Omega}} p_{0}(x) \\
& \equiv p^{+}<+\infty \quad \text { in } \bar{\Omega} .
\end{aligned}
$$

Let $\left\{p_{\varepsilon}\right\}_{(\varepsilon>0)} \subset \mathfrak{Q}_{p_{0}(\cdot)}^{\varepsilon}$ be a sequence defined by

$$
p_{\varepsilon}(x) \stackrel{\text { def }}{=} p_{0}(x)+\mathbf{d}_{\varepsilon}(x)
$$

where the function $\mathbf{d}_{\varepsilon}$ is such that $\mathbf{d}_{\varepsilon}=o(1)$ as $\varepsilon \rightarrow 0$. The asymptotic behavior of $\mathbf{d}_{\varepsilon}$ will be specified below. On the space $W^{1, p_{\varepsilon}(\cdot)}(\Omega)$, we define the functional $J^{\varepsilon}: W^{1, p_{\varepsilon}(\cdot)}(\Omega) \rightarrow$ $\mathbb{R} \cup\{+\infty\}$,

$$
\begin{aligned}
& J^{\varepsilon}[u] \\
& \stackrel{\text { def }}{=}\left\{\begin{array}{cl}
\int_{\Omega}\left\{\frac{K_{\varepsilon}(x)}{p_{\varepsilon}(x)}|\nabla u|^{p_{\varepsilon}(x)}+\frac{1}{\sigma(x)}|u|^{\sigma(x)}\right. & \\
\left.-g^{\varepsilon}(x) u\right\} d x & \text { if } u \in W^{1, p_{\varepsilon}(\cdot)}(\Omega), \\
+\infty & \text { otherwise, }
\end{array}\right.
\end{aligned}
$$


where

$$
K^{\varepsilon}(x)= \begin{cases}k_{f} & \text { in } \Omega_{f}^{\varepsilon}, \\ k_{m} \varepsilon^{p_{0}(x)} & \text { in } \Omega_{m}^{\varepsilon},\end{cases}
$$

the function $\sigma$ satisfies conditions (A.3.4) with $\sigma^{-}>2$, (A.3.5), the function $g^{\varepsilon}$ is defined by $g^{\varepsilon}(x) \stackrel{\text { def }}{=} \mathbf{1}_{f}^{\varepsilon}(x) g(x)$, $g \in C(\Omega)$. Here $k_{f}$ and $k_{m}$ are strictly positive constants independent of $\varepsilon$.

Consider the following variational problem:

$$
J^{\varepsilon}\left[u^{\varepsilon}\right] \longrightarrow \min , \quad u^{\varepsilon} \in W_{0}^{1, p_{\varepsilon}(\cdot)}(\Omega) .
$$

We aim to study the asymptotic behavior of $u^{\varepsilon}$ the solution of (88).

To formulate the main result of this section, we introduce some notations. We denote by $\mathrm{u}^{a}=\mathrm{u}^{a}(x, y)$ the unique solution in $W_{\#}^{1, p_{0}(\cdot)}(F)$ of the following cell problem:

$$
\begin{gathered}
\operatorname{div}_{y}\left\{k_{f}\left|\nabla_{y} \mathrm{u}^{a}\right|^{p_{0}(x)-2} \nabla_{y} \mathrm{u}^{a}\right\}=0 \quad \text { in } F, \\
\left(k_{f}\left|\nabla_{y} \mathrm{u}^{a}\right|^{p_{0}(x)-2} \nabla_{y} \mathrm{u}^{a}-\mathbf{a}, \vec{v}\right)=0 \quad \text { on } \partial M, \\
y \longrightarrow \mathrm{u}^{a}(y) Y \text {-periodic, }
\end{gathered}
$$

where $F=Y \backslash \bar{M}, \vec{v}$ is the outward normal vector to $\partial M$, and $\mathbf{a} \in \mathbb{R}^{n}$. We denote by $\mathrm{w}^{\beta}=\mathrm{w}^{\beta}(x, y)$ the unique solution in $W_{\#}^{1, p_{0}(\cdot)}(M)$ of the following cell problem:

$$
\begin{aligned}
& -\operatorname{div}_{y}\left\{k_{m} \mathbf{d}(x)\left|\nabla_{y} \mathrm{w}^{\beta}\right|^{p_{0}(x)-2} \nabla_{y} \mathrm{w}^{\beta}\right\} \\
& +\left|\mathrm{w}^{\beta}\right|^{\sigma(x)-2} \mathrm{w}^{\beta}=0 \quad \text { in } M, \\
& \mathrm{w}^{\beta}(y)=\beta \quad \text { on } \partial M, \\
& y \longrightarrow \mathrm{w}^{\beta}(y) Y \text {-periodic. }
\end{aligned}
$$

Notice that in (89) and (90) $x$ is a parameter. Regularity results for $u^{a}$ and $w^{\beta}$ are thus easily deduced from [33] and [34]. We also introduce the homogenized functional $J_{\text {hom }}$ : $W^{1, p_{0}(\cdot)}(\Omega) \rightarrow \mathbb{R} \cup\{+\infty\}:$

$$
\begin{aligned}
& J_{\text {hom }}[u] \\
& \stackrel{\operatorname{def}}{=}\left\{\begin{array}{cl}
\int_{\Omega}\left\{T(x, \nabla u)+\frac{\rho^{\star}}{\sigma(x)}|u|^{\sigma(x)}\right. \\
\left.+\mathrm{b}(x, u)-g(x) \rho^{\star} u\right\} d x & \text { if } u \in W_{0}^{1, p_{0}(\cdot)}(\Omega), \\
+\infty & \text { otherwise, }
\end{array}\right.
\end{aligned}
$$

the following results hold.

Theorem 24. Let $u^{\varepsilon}$ be a solution of (88). Assume that

$$
\lim _{\varepsilon \rightarrow 0} \varepsilon^{-\mathbf{d}_{\varepsilon}(\cdot)}=\mathbf{d}(\cdot)
$$

uniformly in $\Omega$. Then $u^{\varepsilon}$ converges strongly in $L^{p_{0}(\cdot)}\left(\Omega_{f}^{\varepsilon}\right)$ to $u$ the solution of the variational problem:

$$
J_{\text {hom }}[u] \longrightarrow \min , \quad u \in W_{0}^{1, p_{0}(\cdot)}(\Omega),
$$

where

$$
\begin{gathered}
\rho^{\star} \stackrel{\text { def }}{=} \text { meas } F, \\
T(x, \mathbf{a}) \stackrel{\text { def }}{=} \frac{1}{p_{0}(x)} \int_{F}\left|\nabla_{y} \mathrm{u}^{a}(x, y)-\mathbf{a}\right|^{p_{0}(x)} d y, \\
\mathrm{~b}(x, \beta) \stackrel{\text { def }}{=} \int_{\mathscr{M}}\left\{\frac{1}{p_{0}(x)}\left[\beta \mathrm{w}^{\beta}\left|\mathrm{w}^{\beta}\right|^{\sigma(x)-2}-\left|\mathrm{w}^{\beta}\right|^{\sigma(x)}\right]\right. \\
\left.+\frac{1}{\sigma(x)}\left|\mathrm{w}^{\beta}\right|^{\sigma(x)}\right\} d y .
\end{gathered}
$$

Theorem 25. Let $u^{\varepsilon}$ be a solution of (88). Assume that, for any $x \in \Omega$,

$$
\lim _{\varepsilon \rightarrow 0} \varepsilon^{-\mathbf{d}_{\varepsilon}(x)}=+\infty
$$

Then $u^{\varepsilon}$ converges strongly in $L^{p_{0}(\cdot)}\left(\Omega_{f}^{\varepsilon}\right)$ to $u$ the solution of the variational problem (93), where $\rho^{\star}$ and the function $T(x, \mathbf{a})$ are given in (94), (95), and

$$
\mathrm{b}(x, \beta) \stackrel{\text { def }}{=} \frac{\text { meas } M}{\sigma(x)}|\beta|^{\sigma(x)} .
$$

Theorem 26. Let $u^{\varepsilon}$ be a solution of (88). Assume that, for any $x \in \Omega$,

$$
\lim _{\varepsilon \rightarrow 0} \varepsilon^{-\mathbf{d}_{\varepsilon}(x)}=0
$$

Then $u^{\varepsilon}$ converges strongly in $L^{p_{0}(\cdot)}\left(\Omega_{f}^{\varepsilon}\right)$ to $u$ the solution of the variational problem (93), where $\rho^{\star}$ and the function $T(x, \mathbf{a})$ are given in (94), (95), and $\mathrm{b}(x, \beta)=0$.

Remark 27. Notice that if $K_{\varepsilon}(x)=\mathbf{1}_{f}^{\varepsilon} k_{f}+\mathbf{1}_{m}^{\varepsilon} k_{m} \varepsilon^{p_{\varepsilon}(x)}$ then Theorem 24 holds true with $\mathbf{d}(x) \equiv 1$.

\section{Homogenization of a Class of Quasilinear Parabolic Equations with Nonstandard Growth}

8.1. Statement of the Problem and Assumptions. In this section, we describe a mesoscopic double porosity model in a periodic fractured medium. We consider a reservoir $\Omega$ C $\mathbb{R}^{n}(n \geq 2)$ to be a bounded connected domain with a periodic structure. More precisely, we will scale this periodic structure by a parameter $\varepsilon$ which represents the ratio of the cell size to the size of the whole region $\Omega$, and we will assume that $\varepsilon$ is a parameter tending to zero. Let $Y \stackrel{\text { def }}{=}$ ] $0,1\left[{ }^{n}\right.$ represent the mesoscopic domain of the basic cell of a fractured porous medium. For the sake of simplicity and without loss of generality, we assume that $Y$ is made up of two homogeneous porous media $M \Subset Y$ and $F$ corresponding 
to parties of the mesoscopic domain occupied by the matrix block and the fracture, respectively. Thus $Y=M \cup \Gamma_{m, f} \cup F$, where $\Gamma_{m, f}$ denotes the interface between the two media and the subscripts $m$ and $f$ refer to the matrix and fracture, respectively. Let $\Omega_{i}^{\varepsilon}$ with $i=m$ or $f$ denotes the open set filled with the porous medium $i$. Then $\Omega=\Omega_{m}^{\varepsilon} \cup \Gamma_{m, f}^{\varepsilon} \cup \Omega_{f}^{\varepsilon}$, where $\Gamma_{m, f}^{\mathcal{E}}=\partial \Omega_{m}^{\varepsilon} \cap \partial \Omega_{f}^{\varepsilon}$. For the sake of simplicity, we will assume that $\partial \Omega \cap \Omega_{m}^{\varepsilon}=\emptyset$.

Let us introduce the nonstandard growth function used in this section. We assume that a family of continuous functions $p_{\varepsilon}=p_{\varepsilon}(x), \varepsilon>0$, is defined in $\bar{\Omega}$ and satisfies the following conditions:

(A.4.1) functions $p_{\varepsilon}$ are bounded from below such that: $p_{\varepsilon}(x) \geq 2$ in $\bar{\Omega}$;

(A.4.2) the function $p_{\varepsilon}(x)$ satisfies the log-Hölder continuity property;

(A.4.3) the function $\gamma_{\varepsilon}(x) \stackrel{\text { def }}{=} p_{\varepsilon}(x)-2$ converges uniformly to zero in $\bar{\Omega}$.

Now let us introduce the permeability coefficient and the porosity of the porous medium $\Omega$. We set

$$
\begin{aligned}
& k^{\varepsilon}(x)=k_{m} \varepsilon^{2} \mathbf{1}_{m}^{\varepsilon}(x)+k_{f} \mathbf{1}_{f}^{\varepsilon}(x), \\
& \omega^{\varepsilon}(x)=\omega_{m} \mathbf{1}_{m}^{\varepsilon}(x)+\omega_{f} \mathbf{1}_{f}^{\varepsilon}(x),
\end{aligned}
$$

where $k_{f}$ is the permeability or the hydraulic conductivity of fissures, $k_{m}$ is the permeability or the hydraulic conductivity of blocks, $\omega_{f}$ is the porosity of fissures, and $\omega_{m}$ is the porosity of blocks; $\mathbf{1}_{f}^{\varepsilon}=\mathbf{1}_{f}^{\varepsilon}(x)$ and $\mathbf{1}_{m}^{\varepsilon}=\mathbf{1}_{m}^{\varepsilon}(x)$ denote the characteristic functions of the sets $\Omega_{f}^{\varepsilon}$ and $\Omega_{m}^{\varepsilon}$, respectively. Here $0<k_{f}, k_{m}, \omega_{f}, \omega_{m}<+\infty$.

We consider the following initial boundary value problem for the function $u^{\varepsilon}: Q \mapsto \mathbb{R}$ :

$$
\begin{gathered}
\omega^{\varepsilon}(x) \frac{\partial u^{\varepsilon}}{\partial t}-\operatorname{div}\left(k^{\varepsilon}(x) \nabla u^{\varepsilon}\left|\nabla u^{\varepsilon}\right|^{p_{\varepsilon}(x)-2}\right)=g(t, x) \text { in } Q, \\
u^{\varepsilon}=0 \quad \text { on }(0, T) \times \partial \Omega, \\
u^{\varepsilon}(0, x)=u_{0}(x) \quad \text { in } \Omega,
\end{gathered}
$$

where $Q$ denotes the cylinder $(0, T) \times \Omega, T>0$ is given, and $g, u_{0}$ are given functions.

For simplicity and without loss of generality, we restrict the presentation to a homogeneous Dirichlet boundary condition on $\partial \Omega$, but it is easy to see that all results also hold for other boundary conditions.

Throughout the section, $C$ will denote a generic positive constant, independent of $\varepsilon$, and may take different values for different occurrences.

8.2. Preliminary Results. The goal of this section is to obtain $a$ priori estimates for the solution $u^{\varepsilon}$ of problem (101). We start by formulating the existence and uniqueness result for (101). It is given by the following theorem (see [35]).
Theorem 28. Let $g \in C\left(0, T ; L^{2}(\Omega)\right)$ and $u_{0} \in H^{2}(\Omega)$. Then, for any $\varepsilon>0$, there exists a unique solution $u^{\varepsilon}=$ $u^{\varepsilon}(t, x)$ of the boundary value problem (101) in the space $L^{\infty}\left(0, T ; W^{1, p_{\varepsilon}(\cdot)}(\Omega)\right)$. Furthermore, this solution satisfies the following a priori estimates, for a.e. $t \in(0, T)$ :

$$
\begin{aligned}
& \left\|u^{\varepsilon}(t)\right\|_{L^{2}(\Omega)}^{2}+\int_{0}^{t} d t \int_{\Omega}\left|\frac{\partial u^{\varepsilon}}{\partial t}\right|^{2} d x+\int_{\Omega} k^{\varepsilon}(x)\left|\nabla u^{\varepsilon}\right|^{p_{\varepsilon}(x) d x} \\
& \quad \leq C \\
& \left\|u^{\varepsilon}(t+\delta t)-u^{\varepsilon}(t)\right\|_{L^{2}(\Omega)} \leq C(\delta t)^{\kappa} \quad \text { with } 0<\kappa<1
\end{aligned}
$$

where $\delta t>0$ is a time step which tends to zero.

We study the asymptotic behavior of the solution $u^{\varepsilon}$ of problem (101) as $\varepsilon \rightarrow 0$. For this it is convenient to introduce the following notation:

$$
u^{\varepsilon}= \begin{cases}\rho^{\varepsilon} & \text { in } \Omega_{f}^{\varepsilon}, \\ \sigma^{\varepsilon} & \text { in } \Omega_{m}^{\varepsilon},\end{cases}
$$

and to rewrite (101) separately in the domains $\Omega_{f}^{\varepsilon}, \Omega_{m}^{\varepsilon}$ with appropriate interface conditions. Namely, in the domain $\Omega_{f}^{\varepsilon}$ (101) reads

$$
\begin{aligned}
& \omega_{f} \frac{\partial \rho^{\varepsilon}}{\partial t}-\operatorname{div}\left(k_{f} \nabla \rho^{\varepsilon}\left|\nabla \rho^{\varepsilon}\right|^{p_{\varepsilon}(x)-2}\right) \\
& =g(t, x) \quad \operatorname{in}(0, T) \times \Omega_{f}^{\varepsilon}, \\
& k_{f} \nabla \rho^{\varepsilon}\left|\nabla \rho^{\varepsilon}\right|^{p_{\varepsilon}(x)-2} \cdot \vec{v} \\
& =k_{m} \varepsilon^{p_{\varepsilon}(x)} \nabla \sigma^{\varepsilon}\left|\nabla \sigma^{\varepsilon}\right|^{p_{\varepsilon}(x)-2} \cdot \vec{v} \quad \text { on }(0, T) \times \Gamma_{m, f}^{\varepsilon}, \\
& \rho^{\varepsilon}=0 \quad \text { on }(0, T) \times \partial \Omega, \\
& \rho^{\varepsilon}(0, x)=u_{0}(x) \quad \text { in } \Omega_{f}^{\varepsilon},
\end{aligned}
$$

where $\vec{v}$ is the outward normal vector to $\Gamma_{m f}^{\varepsilon}$. In the domain $\Omega_{m}^{\varepsilon}(101)$ reads

$$
\begin{gathered}
\omega_{m} \frac{\partial \sigma^{\varepsilon}}{\partial t}-\operatorname{div}\left(k_{m} \varepsilon^{2} \nabla \sigma^{\varepsilon}\left|\nabla \sigma^{\varepsilon}\right|^{p_{\varepsilon}(x)-2}\right) \\
=g(t, x) \quad \text { in }(0, T) \times \Omega_{m}^{\varepsilon}, \\
\sigma^{\varepsilon}=\rho^{\varepsilon} \quad \text { on }(0, T) \times \Gamma_{m f}^{\varepsilon}, \\
\sigma^{\varepsilon}(0, x)=u_{0}(x) \quad \text { in } \Omega_{m}^{\varepsilon} .
\end{gathered}
$$

To establish some preliminary compactness result, first we notice that the a priori estimate (102), conditions (A.4.1), (A.4.3) along with (110), and inequalities (106), and (107) imply the bound for a.e. $t \in(0, T)$ :

$$
\begin{aligned}
& \left\|u^{\varepsilon}(t)\right\|_{L^{2}(\Omega)}+\int_{0}^{t} d t \int_{\Omega}\left|\frac{\partial u^{\varepsilon}}{\partial t}\right|^{2} d x \\
& \quad+\int_{\Omega_{f}^{\varepsilon}}\left|\nabla u^{\varepsilon}\right|^{2} d x+\varepsilon^{2} \int_{\Omega_{m}^{\varepsilon}}\left|\nabla u^{\varepsilon}\right|^{2} d x \leq C .
\end{aligned}
$$


Therefore, from (105), (106) and (107), we have for a.e. $t \in$ $(0, T)$ :

$$
\begin{gathered}
\left\|\rho^{\varepsilon}(t)\right\|_{L^{2}\left(\Omega_{f}^{\varepsilon}\right)}+\int_{0}^{t} d t \int_{\Omega_{f}^{\varepsilon}}\left|\frac{\partial \rho^{\varepsilon}}{\partial t}\right|^{2} d x+\int_{\Omega_{f}^{\varepsilon}}\left|\nabla \rho^{\varepsilon}\right|^{2} d x \leq C, \\
\left\|\sigma^{\varepsilon}(t)\right\|_{L^{2}\left(\Omega_{m}^{\varepsilon}\right)}+\int_{0}^{t} d t \int_{\Omega_{m}^{\varepsilon}}\left|\frac{\partial \sigma^{\varepsilon}}{\partial t}\right|^{2} d x+\varepsilon^{2} \int_{\Omega_{m}^{\varepsilon}}\left|\nabla \sigma^{\varepsilon}\right|^{2} d x \leq C .
\end{gathered}
$$

Now using the bounds (108), along with the extension result [30], it is easy to prove the following compactness result.

Lemma 29. Let $u^{\varepsilon}=\left\langle\rho^{\varepsilon}, \sigma^{\varepsilon}\right\rangle$ be the solution of problem (101). Then there exists a subsequence, still denoted by $\left\{u^{\varepsilon}\right\}_{\varepsilon>0}$, and functions $u_{f}=u_{f}(t, x), v_{f}=v_{f}(x, y), u_{m}=u_{m}(t, x, y)$ such that

(a.1) $u_{f} \in H^{1}\left(0, T ; L^{2}(\Omega)\right) \cap L^{\infty}\left(0, T ; H^{1}(\Omega)\right), v_{f} \in$ $L^{2}\left(\Omega ; H_{\#}^{1}(F) \backslash \mathbb{R}\right)$,

(a.2) $u_{m} \in H^{1}\left(0, T ; L^{2}(\Omega \times Y)\right) \cap L^{\infty}\left(0, T ; L^{2}\left(\Omega ; H^{1}(M)\right)\right)$, with $u_{m}(t, x, y)=u_{f}(t, x)$ for $y \in \partial M$,

(b) for any $t \in(0, T), \rho^{\varepsilon} \stackrel{2 s}{\longrightarrow} u_{f}$ and $\sigma^{\varepsilon} \stackrel{2 s}{\longrightarrow} u_{m}$,

(c) for any $\varphi \in L^{\infty}\left(0, T ; L^{2}\left(\Omega ; C_{\#}(Y)\right)\right)$,

$$
\begin{aligned}
& \int_{0}^{T} d t \int_{\Omega_{f}^{\varepsilon}} \frac{\partial u^{\varepsilon}}{\partial t} \varphi\left(t, x, \frac{x}{\varepsilon}\right) d x \\
& \longrightarrow \int_{0}^{T} d t \int_{\Omega \times F} \frac{\partial u_{f}}{\partial t} \varphi(t, x, y) d x d y, \\
& \int_{0}^{T} d t \int_{\Omega_{m}^{\varepsilon}} \frac{\partial u^{\varepsilon}}{\partial t} \varphi\left(t, x, \frac{x}{\varepsilon}\right) d x \\
& \longrightarrow \int_{0}^{T} d t \int_{\Omega \times M} \frac{\partial u_{m}}{\partial t} \varphi(t, x, y) d x d y,
\end{aligned}
$$

(d) for a.e. $t \in(0, T), \nabla \rho^{\varepsilon} \stackrel{2 s}{\longrightarrow}\left(\nabla_{x} u_{f}+\nabla_{y} v_{f}\right) \mathbf{1}_{f}(y)$ and $\nabla \sigma^{\varepsilon} \stackrel{2 s}{\rightarrow} \nabla_{y} u_{m} \mathbf{1}_{m}(y)$.

8.3. Homogenization Results. In this section, we formulate the main results of the section. We give homogenization results for the problem (101). The convergence of the homogenization process is obtained by combining the technique of two-scale convergence and the variational homogenization method (see, e.g., $[9,16,27]$ and the references therein).

The idea of the proof is the following. First we will reduce our parabolic problem to an elliptic one depending on the time variable as a parameter. Then we introduce a functional corresponding to this elliptic problem and study the minimization problem for it in the limit of small $\varepsilon$. Then we obtain the limit functional corresponding to the homogenized problem. Regarding the variational technique, it is worth to mention one trick used in the paper. In order to obtain the lower bound for the original functional, we first replace the original exponent $p_{\varepsilon}(x)$ by a new one $p_{0}=2$ and consider the corresponding family of auxiliary functionals. Then the lower semicontinuity property of convex functionals with respect to the two-scale convergence implies the desired inequality. Finally, it is not difficult to show that the limit functional for the auxiliary family does not exceed the limiting functional for the original one.

Now we are in position to formulate the first homogenization result of the section.

Theorem 30. Let $u^{\varepsilon}=u^{\varepsilon}(t, x)$ be the solution of the boundary value problem (101) and let conditions (A.4.1)-(A.4.3) be satisfied. Moreover, we assume that there exists a function $\alpha \in$ $C(\bar{\Omega})$ such that uniformly in $x \in \bar{\Omega}$,

$$
\lim _{\varepsilon \rightarrow 0} \varepsilon^{-\gamma_{\varepsilon}(x)}=\alpha(x) .
$$

Then, for a.e. $t \in(0, T), u^{\varepsilon}$ two-scale converges to $u^{*} \in$ $L^{2}((0, T) \times \Omega \times Y)$ such that

$$
u^{*}(t, x, y)= \begin{cases}u_{f}(t, x) & \text { in } Q \times F \\ u_{m}(t, x, y) & \text { in } Q \times M\end{cases}
$$

where the couple $\left(u_{f}, u_{m}\right) \in L^{2}\left(0, T ; H^{1}(\Omega)\right) \times L^{\infty}(0, T$; $\left.L^{2}\left(\Omega ; H^{1}(M)\right)\right)$ is the unique solution of the homogenized problem

$$
\begin{gathered}
\omega_{f}|F| \frac{\partial u_{f}}{\partial t}-\operatorname{div}_{x}\left(K^{*} \nabla_{x} u_{f}\right)=S\left(x, u_{m}\right) \quad \text { in } Q \\
u_{f}=0 \text { on }(0, T) \times \partial \Omega, \quad u_{f}(0, x)=u_{0}(x) \quad \text { in } \Omega, \\
\omega_{m} \frac{\partial u_{m}}{\partial t}-\widetilde{K}^{*}(x) \Delta_{y} u_{m}=g(t, x) \quad \text { in } Q \times M, \\
u_{m}(t, x, y)=u_{f}(t, x) \quad \text { on } Q \times \partial M, \\
u_{m}(0, x, y)=u_{0}(x) \quad \text { in } \Omega \times M,
\end{gathered}
$$

where $|F|$ is the measure of the set $F$ and $K^{*}=\left\{k_{i j}^{*}\right\}$ is the homogenized permeability tensor defined by

$$
k_{i j}^{*}=k_{f} \int_{F}\left(\vec{e}_{i}+\nabla_{y} w_{i}\right) \cdot\left(\vec{e}_{j}+\nabla_{y} w_{j}\right) d y
$$

with $\left\{\vec{e}_{1}, \vec{e}_{2}, \ldots, \vec{e}_{n}\right\}$ the canonical basis of $\mathbb{R}^{n}$ and $w_{i}$ being the unique solution in $H_{\#}^{1}(F) \backslash \mathbb{R}$ of

$$
\begin{gathered}
-k_{f} \Delta_{y} w_{i}=0 \quad \text { in } F, \\
\left(\vec{e}_{i}+\nabla_{y} w_{i}\right) \cdot \vec{v}=0 \quad \text { on } \partial M, \\
y \longrightarrow w_{i}(x, y) \quad Y \text {-periodic, }
\end{gathered}
$$

where $\vec{v}=\vec{v}(y)$ is the outer normal vector at $\partial M$, and the coefficient $\widetilde{K}^{*}$ in the local problem is given by

$$
\widetilde{K}^{*}(x)=\alpha(x) k_{m},
$$


and the effective source term $\mathrm{S}\left(x, u_{m}\right)$ is given by

$$
\mathrm{S}\left(x, u_{m}\right)=|F| g(t, x)-\widetilde{K}^{*}(x) \int_{\partial M}\left(\nabla_{y} u_{m} \cdot \vec{\nu}\right) d s_{y} .
$$

Remark 31. The source term which appears in the righthand side of the first equation in (112) is well defined, since $u_{m} \in L^{\infty}\left(0, T ; L^{2}\left(\Omega ; H^{1}(M)\right)\right)$, and it follows from the third equation of (112) that

$$
-\Delta_{y} u_{m} \in L^{2}\left(0, T ; H^{-1 / 2}(\partial M)\right),
$$

which allows one to define $\left(\nabla_{y} u_{m} \cdot \vec{v}\right)$ as an element of $L^{2}\left(0, T ; H^{-1 / 2}(\partial M)\right)$.

The Scheme of the Proof of Theorem 30 Is as Follows. We consider our parabolic boundary value problem (101) as an elliptic one depending on the time variable $t$ as a parameter. Namely, we consider the following boundary value problem, for a.e. $t \in] 0, T[$,

$$
\begin{gathered}
-\operatorname{div}\left(k^{\varepsilon}(x) \nabla u^{\varepsilon}\left|\nabla u^{\varepsilon}\right|^{p_{\varepsilon}(x)-2}\right)=G^{\varepsilon} \quad \text { in } Q, \\
\left.u^{\varepsilon}=0 \quad \text { on }\right] 0, T[\times \partial \Omega,
\end{gathered}
$$

where the function $G^{\varepsilon}$,

$$
G^{\varepsilon}=G^{\varepsilon}(t, x) \stackrel{\text { def }}{=} g(t, x)-\omega^{\varepsilon}(x) \frac{\partial u^{\varepsilon}}{\partial t}(t, x),
$$

is considered as a given function. Then, for any $\Delta_{t} \subset[0, T]$, $u^{\varepsilon}$ minimizes the functional

$$
J^{\varepsilon}[u] \stackrel{\text { def }}{=} \int_{\Delta_{t}} d t \int_{\Omega}\left\{\frac{k^{\varepsilon}(x)}{p_{\varepsilon}(x)}|\nabla u|^{p_{\varepsilon}(x)}-G^{\varepsilon} u\right\} d x
$$

over $u \in L^{\infty}\left(0, T ; W^{1, p_{\varepsilon}(\cdot)}(\Omega)\right)$. Using the two-scale convergence arguments, we obtain the "lim sup"-inequality for the functional $J^{\varepsilon}$. The "lim inf"-inequality is obtained in two steps. First, we introduce the auxiliary functional

$$
\begin{aligned}
& \widetilde{J}^{\varepsilon}[u] \stackrel{\text { def }}{=} \int_{\Delta_{t}} d t \int_{\Omega}\left\{\frac{\widetilde{k}^{\varepsilon}(x)}{p_{\varepsilon}(x)}|\nabla u|^{2}-G^{\varepsilon} u\right\} d x \\
& \text { with } \widetilde{k}^{\varepsilon}(x)=\alpha(x) k_{m} \varepsilon^{2} \mathbf{1}_{m}^{\varepsilon}(x)+k_{f} \mathbf{1}_{f}^{\varepsilon}(x)
\end{aligned}
$$

and obtain the inequality

$$
\varliminf_{\varepsilon \rightarrow 0} \widetilde{J}^{\varepsilon}\left[u^{\varepsilon}\right] \geq J_{\text {hom }}\left[u_{f}, u_{m}\right]
$$

where

$$
\begin{aligned}
& J_{\text {hom }}\left[u_{f}, u_{m}\right] \\
& =\int_{\Delta_{t}} d t \int_{\Omega}\left\{\frac{1}{2}\left(K^{*} \nabla_{x} u_{f} \cdot \nabla_{x} u_{f}\right)\right. \\
& \left.\quad-|F|\left(g(t, x)-\omega_{f} \frac{\partial u_{f}}{\partial t}\right) u_{f}\right\} d x \\
& +\int_{\Delta_{t}} d t \int_{\Omega \times M}\left\{\alpha(x) \frac{k_{m}}{2}\left|\nabla_{y} u_{m}\right|^{2}\right. \\
& \left.\quad-\left(g(t, x)-\omega_{m} \frac{\partial u_{m}}{\partial t}\right) u_{m}\right\} d x d y .
\end{aligned}
$$

Then in the second step, we obtain the desired "lim inf"inequality for the initial functional. This completes the proof of Theorem 30.

The macroscopic model corresponding to the second situation is given by the following convergence result.

Theorem 32. Let $u^{\varepsilon}=\left\langle\rho^{\varepsilon}, \sigma^{\varepsilon}\right\rangle$ be the solution of the boundary value problem (101) and let conditions (A.4.1)-(A.4.3) be satisfied. Moreover, we assume that for any $x \in \bar{\Omega}$,

$$
\lim _{\varepsilon \rightarrow 0} \varepsilon^{-\gamma_{\varepsilon}(x)}=+\infty \text {. }
$$

Then, for a.e. $t \in(0, T)$, $u^{\varepsilon}$ converges in $L^{2}(\Omega)$, as $\varepsilon \rightarrow 0$, to $u^{0} \in L^{2}\left(0, T ; H^{1}(\Omega)\right)$, the solution of

$$
\begin{gathered}
\left(\omega_{f}|F|+\omega_{m}|M|\right) \frac{\partial u^{0}}{\partial t}-\operatorname{div}_{x}\left(K^{*} \nabla_{x} u^{0}\right)=g(t, x) \quad \text { in } Q, \\
u^{0}=0 \quad \text { on }(0, T) \times \partial \Omega, \quad u^{0}(0, x)=u_{0}(x) \quad \text { in } \Omega,
\end{gathered}
$$

where $K^{*}=\left\{k_{i j}^{*}\right\}$ is the homogenized permeability tensor defined in (113)-(114).

The proof of Theorem 32 is similar to that of Theorem 30.

Remark 33. Notice that the structure of the limit problem depends crucially on the rate of convergence of $\left(p_{\varepsilon}(\cdot)-2\right)$ to zero. The critical rate of convergence is

$$
\left(p_{\varepsilon}(\cdot)-2\right) \sim \frac{1}{|\ln \varepsilon|} .
$$

More precisely, if $\overline{\lim }_{\varepsilon \rightarrow 0}|\ln \varepsilon|\left(p_{\varepsilon}(x)-2\right)<+\infty$, then the limit model is of a double porosity type. If $\lim _{\varepsilon \rightarrow 0}|\ln \varepsilon|\left(p_{\varepsilon}(x)-2\right)=+\infty$, then in the limit we obtain a single porosity model.

The proofs of Theorems 30 and 32 are given in [21].

\section{References}

[1] S. N. Antontsev and S. I. Shmarev, "Elliptic equations with anisotropic nonlinearity and nonstandard growth conditions," 
in Handbook of Differential Equations. Stationary Partial Differential Equations, vol. 3, chapter 1, pp. 1-100, Elsevier, 2006.

[2] S. Antontsev and S. Shmarev, "Elliptic equations and systems with nonstandard growth conditions: existence, uniqueness and localization properties of solutions," Nonlinear Analysis. Theory, Methods \& Applications A, vol. 65, no. 4, pp. 728-761, 2006.

[3] X.-L. Fan and Q.-H. Zhang, "Existence of solutions for $p(x)$ Laplacian Dirichlet problem," Nonlinear Analysis. Theory, Methods \& Applications A, vol. 52, no. 8, pp. 1843-1852, 2003.

[4] H. Hudzik, “On generalized Orlicz-Sobolev space," Functiones et Approximatio Commentarii Mathematici, vol. 4, pp. 37-51, 1976.

[5] O. Kováčik and J. Rákosník, "On spaces $L^{p(x)}$ and $W^{k, p(x), "}$ Czechoslovak Mathematical Journal, vol. 41, no. 4, pp. 592-618, 1991.

[6] E. Acerbi and G. Mingione, "Regularity results for a class of functionals with non-standard growth," Archive for Rational Mechanics and Analysis, vol. 156, no. 2, pp. 121-140, 2001.

[7] J. Musielak, Orlicz Spaces and Modular Spaces, vol. 1034 of Lecture Notes in Mathematics, Springer, Berlin, Germany, 1983.

[8] I. I. Šarapudinov, "The topology of the space $L^{p^{(t)}}([0,1])$," Matematicheskie Zametki, vol. 26, pp. 613-632, 1979.

[9] A. Braides and A. Defranceschi, Homogenization of Multiple Integrals, vol. 12 of Oxford Lecture Series in Mathematics and its Applications, The Clarendon Press/Oxford University Press, 1998.

[10] G. Dal Maso, An Introduction to Г-Convergence, Birkhäuser, Boston, Mass, USA, 1993.

[11] S. M. Kozlov, "Geometric aspects of averaging," Russian Mathematical Surveys, vol. 44, pp. 79-120, 1989.

[12] V. V. Zhikov, "Lavrentiev effect and the averaging of nonlinear variational problems," Differential Equation, vol. 27, pp. 32-39, 1991.

[13] V. V. Zhikov, "On passage to the limit in nonlinear variational problems," Matematicheskiu Sbornik, vol. 183, no. 8, pp. 47-84, 1992.

[14] V. Zhikov, "Lavrentiev phenomenon and homogenization for some variational problems," Comptes Rendus de l'Académie des Sciences, vol. 316, no. 5, pp. 435-439, 1993.

[15] V. V. Zhikov, “On Lavrentiev's phenomenon," Russian Journal of Mathematical Physics, vol. 3, no. 2, pp. 249-269, 1995.

[16] V. V. Jikov, S. M. Kozlov, and O. A. Oleĭnik, Homogenization of Differential Operators and Integral Functionals, Springer, New York, NY, USA, 1994.

[17] B. Amaziane, S. Antontsev, and L. Pankratov, "Homogenization of a class of nonlinear elliptic equations with nonstandard growth," Comptes Rendus, vol. 335, no. 3, pp. 138-143, 2007.

[18] B. Amaziane, S. Antontsev, L. Pankratov, and A. Piatnitski, " $\Gamma$-convergence and homogenization of functionals in Sobolev spaces with variable exponents," Journal of Mathematical Analysis and Applications, vol. 342, no. 2, pp. 1192-1202, 2008.

[19] B. Amaziane, S. Antontsev, L. Pankratov, and A. Piatnitski, "Homogenization of $p$-Laplacian in perforated domain," Annales de l'Institut Henri Poincaré, vol. 26, no. 6, pp. 2457-2479, 2009.

[20] B. Amaziane, L. Pankratov, and A. Piatnitski, "Homogenization of a class of quasilinear elliptic equations in high-contrast fissured media," Proceedings of the Royal Society of Edinburgh A, vol. 136, no. 6, pp. 1131-1155, 2006.
[21] B. Amaziane, L. Pankratov, and A. Piatnitski, "Nonlinear flow through double porosity media in variable exponent Sobolev spaces," Nonlinear Analysis. Real World Applications, vol. 10, no. 4, pp. 2521-2530, 2009.

[22] B. Amaziane, L. Pankratov, and A. Piatnitski, "Homogenization of variational functionals with nonstandard growth in perforated domains," Networks and Heterogeneous Media, vol. 5, no. 2, pp. 189-215, 2010.

[23] B. Amaziane, L. Pankratov, and V. Prytula, "Nonlocal effects in homogenization of $p_{\epsilon}(x)$-Laplacian in perforated domains," Nonlinear Analysis. Theory, Methods \& Applications A, vol. 71, no. 9, pp. 4078-4097, 2009.

[24] B. Amaziane, L. Pankratov, and V. V. Pritula, "Homogenization of $p_{\varepsilon}(x)$-Laplacian in perforated domains with a nonlocal transmission condition," Comptes Rendus Mécanique, vol. 337, pp. 173-178, 2009.

[25] C. Choquet and L. Pankratov, "Nonlinear double porosity models with non-standard growth," Comptes Rendus Mécanique, vol. 337, no. 9-10, pp. 659-666, 2009.

[26] C. Choquet and L. Pankratov, "Homogenization of a class of quasilinear elliptic equations with non-standard growth in high-contrast media," Proceedings of the Royal Society of Edinburgh A, vol. 140, no. 3, pp. 495-539, 2010.

[27] V. A. Marchenko and E. Y. Khruslov, Homogenization of Partial Differential Equations, Birkhäuser, Boston, Mass, USA, 2006.

[28] A. A. Kovalevskiü, "Conditions for $\Gamma$-convergence and homogenization of integral functionals with different domains of the definition," Doklady Akademii Nauk Ukrainskoj SSR, no. 4, pp. $5-8,1991$.

[29] G. Allaire, "Homogenization and two-scale convergence," SIAM Journal on Mathematical Analysis, vol. 23, no. 6, pp. 1482-1518, 1992.

[30] E. Acerbi, V. C. Piat, G. Dal Maso, and D. Percivale, "An extension theorem from connected sets, and homogenization in general periodic domains," Nonlinear Analysis. Theory, Methods \& Applications A, vol. 18, no. 5, pp. 481-496, 1992.

[31] D. Cioranescu and J. S. J. Paulin, Homogenization of Reticulated Structures, vol. 136 of Applied Mathematical Sciences, Springer, New York, NY, USA, 1999.

[32] L. Pankratov and A. Piatnitski, "Nonlinear "double porosity" type model," Comptes Rendus Mathématique, vol. 334, no. 5, pp. 435-440, 2002.

[33] L. C. Evans, "A new proof of local $C^{1, \alpha}$ regularity for solutions of certain degenerate elliptic p.d.e," Journal of Differential Equations, vol. 45, no. 3, pp. 356-373, 1982.

[34] P. Tolksdorf, "Regularity for a more general class of quasilinear elliptic equations," Journal of Differential Equations, vol. 51, no. 1, pp. 126-150, 1984.

[35] S. Antontsev and S. Shmarev, "Parabolic equations with anisotropic nonstandard growth conditions," in Free Boundary Problems. Theory and Applications, vol. 154 of International Series of Numerical Mathematics, pp. 33-44, Birkhäuser, Basel, Switzerland, 2007. 


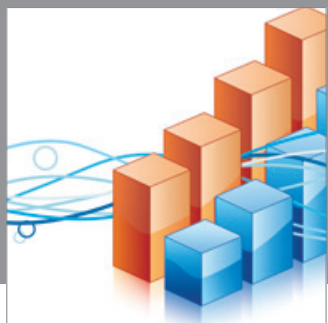

Advances in

Operations Research

mansans

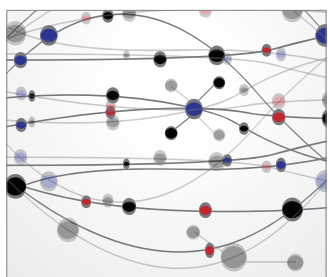

The Scientific World Journal
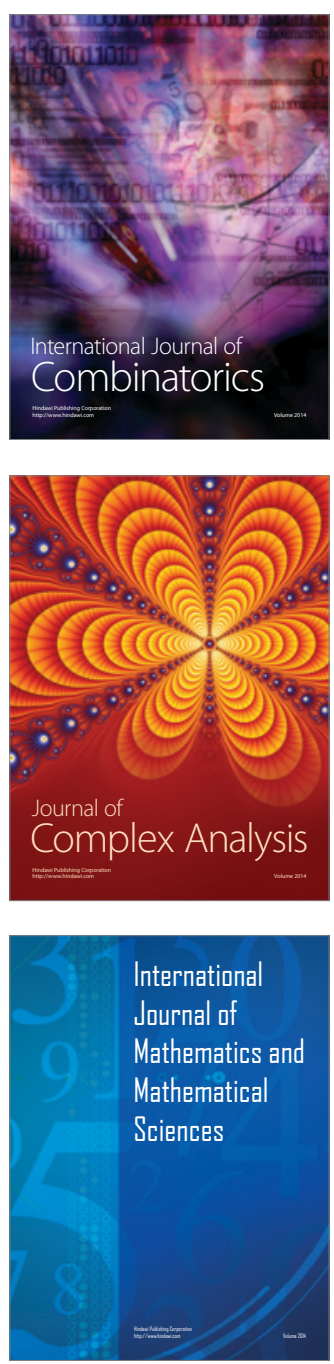
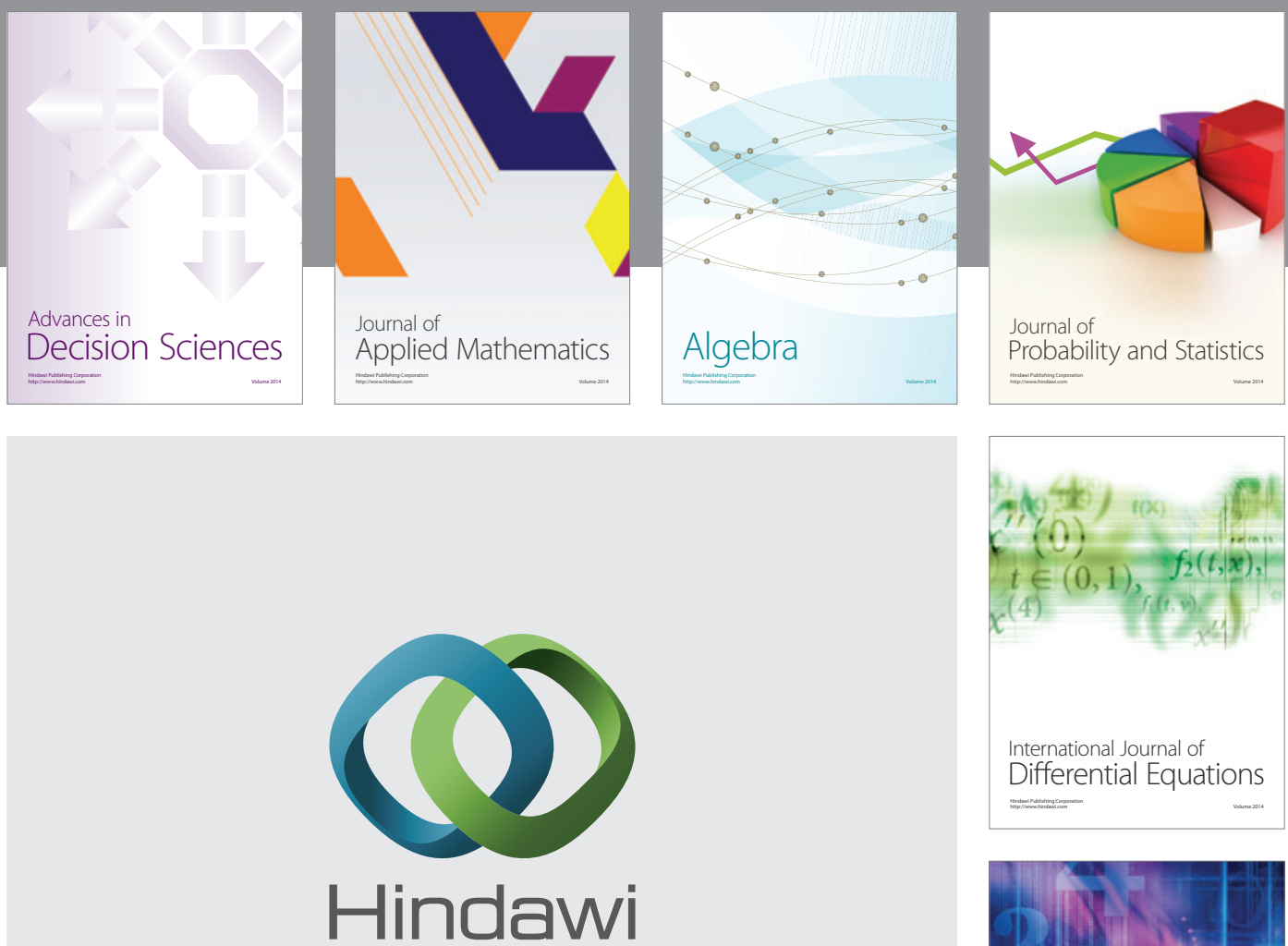

Submit your manuscripts at http://www.hindawi.com
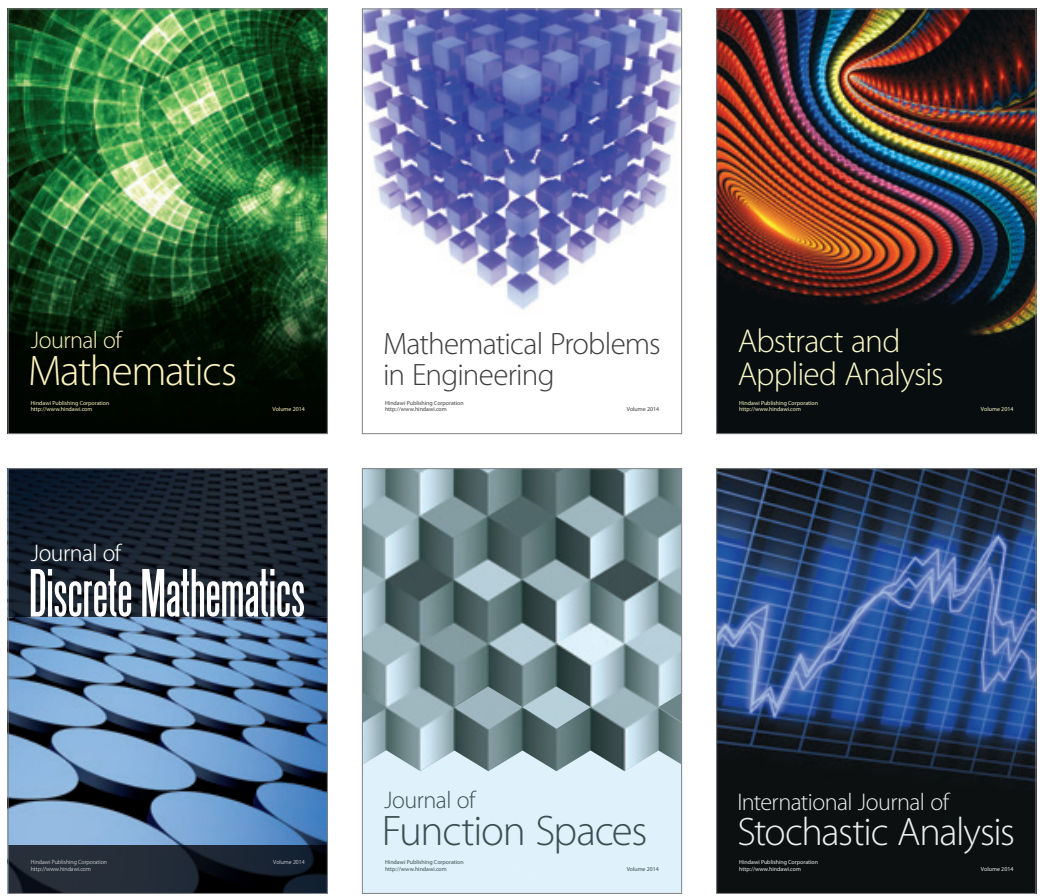

Journal of

Function Spaces

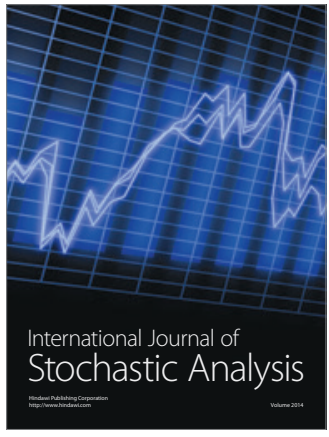

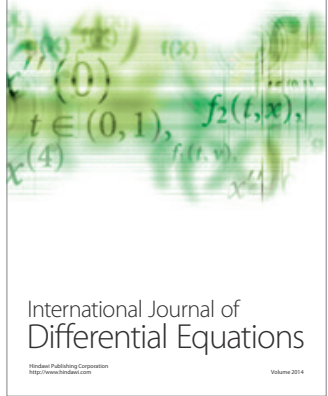
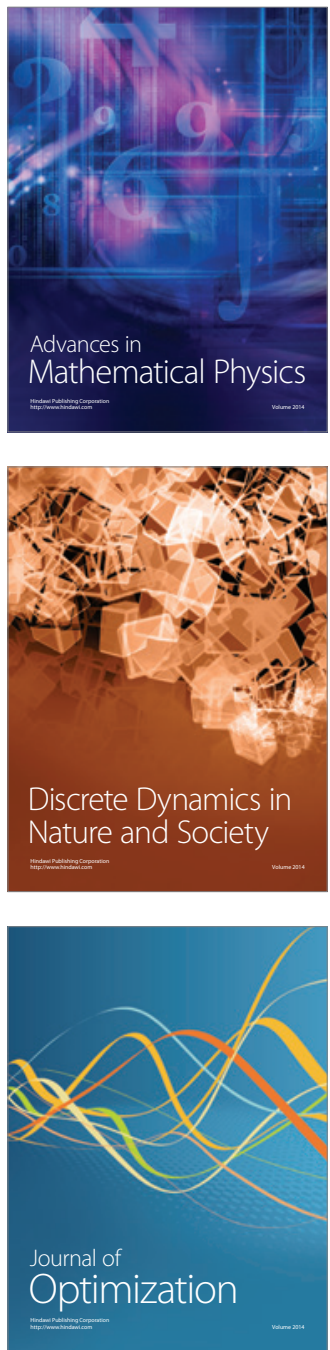